\title{
Computing nucleon charges with highly improved staggered quarks
}

\author{
Yin Lin (林)㻆) $\odot,{ }^{1,2, *}$ Aaron S. Meyer, ${ }^{3, \dagger}$ Steven Gottlieb, ${ }^{4}$ Ciaran Hughes, ${ }^{2}$ Andreas S. Kronfeld, \\ James N. Simone, ${ }^{2}$ and Alexei Strelchenko ${ }^{2}$
}

(Fermilab Lattice Collaboration)

${ }^{1}$ Enrico Fermi Institute, University of Chicago, Chicago, Illinois 60637, USA

${ }^{2}$ Fermi National Accelerator Laboratory, Batavia, Illinois 60510, USA

${ }^{3}$ Brookhaven National Laboratory, Upton, New York 11973, USA

${ }^{4}$ Department of Physics, Indiana University, Bloomington, Indiana 47405, USA

(Received 27 October 2020; accepted 17 February 2021; published 23 March 2021)

\begin{abstract}
This work continues our program of lattice-QCD baryon physics using staggered fermions for both the sea and the valence quarks. We present a proof-of-concept study that demonstrates, for the first time, how to calculate baryon matrix elements using staggered quarks for the valence sector. We show how to relate the representations of the continuum staggered flavor-taste group $\mathrm{SU}(8)_{\mathrm{FT}}$ to those of the discrete lattice symmetry group. The resulting calculations yield the normalization factors relating staggered baryon matrix elements to their physical counterparts. We verify this methodology by calculating the isovector vector and axial-vector charges $g_{V}$ and $g_{A}$. We use a single ensemble from the MILC Collaboration with $2+1+1$ flavors of sea quark, lattice spacing $a \approx 0.12 \mathrm{fm}$, and a pion mass $M_{\pi} \approx 305 \mathrm{MeV}$. On this ensemble, we find results consistent with expectations from current conservation and neutron beta decay. Thus, this work demonstrates how highly improved staggered quarks can be used for precision calculations of baryon properties and, in particular, the isovector nucleon charges.
\end{abstract}

DOI: 10.1103/PhysRevD.103.054510

\section{INTRODUCTION}

Accurate first-principles calculations of nuclear cross sections are an important objective in the particle physics community. In particular, heavy nuclei, such as ${ }^{12} \mathrm{C}$ and ${ }^{40} \mathrm{Ar}$, are used as targets in neutrino-scattering and darkmatter detection experiments. In calculations of cross sections, a necessary component is the modeling of nuclei as collections of nucleons, opening up an opportunity for lattice QCD [1]. At the quasielastic peak, for example, the electromagnetic and axial-vector form factors of the nucleon, which characterize the electric charge and spin distribution within the nucleon, are key ingredients. Such form factors can be obtained from first principles with lattice QCD. These hadronic inputs, however, remain one

\footnotetext{
yin01@uchicago.edu

asmeyer.physics@gmail.com

Present address: UC Berkeley and Lawrence Berkeley National Laboratory, Berkeley, CA 94720, USA.

*ask@fnal.gov

Published by the American Physical Society under the terms of the Creative Commons Attribution 4.0 International license. Further distribution of this work must maintain attribution to the author(s) and the published article's title, journal citation, and DOI. Funded by SCOAP ${ }^{3}$.
}

of the largest sources of systematic error as the experimental precision on these cross sections continues to improve [2-4].

The electromagnetic form factors have been extracted precisely from high statistics electron-nucleon scattering experiments $[5,6]$. At zero momentum transfer, the proton's electric form factor becomes the total electric charge $g_{V}=1$, and the slope at the origin is related to the charge radius. Recently, experiments that make use of the Lamb shift of muonic hydrogen report significantly smaller proton radii than those measured via scattering [7]. (For recent reviews of the proton radius puzzle, see Refs. $[8,9]$.) In addition, a recent reanalysis has demonstrated that the vector form factors at intermediate $Q^{2}$ also exhibit tensions outside of their quoted uncertainties [10]. These disagreements could benefit from better knowledge of the Standard Model predictions, which necessitates using lattice QCD to calculate the form factor.

In comparison, the nucleon axial-vector form factor is much less constrained from experimental data. A recent reanalysis [11] of the deuterium bubble-chamber data found greater uncertainties than previously assumed. Again, lattice QCD can be illuminating here, computing the axial-vector form factor from first principles as an independent check on the form factor extracted from 
experimental data. At zero momentum transfer, the axialvector form factor gives the so-called nucleon axial charge $g_{A}=1.2756(13)$, which has been measured precisely in neutron beta decay [12]. Thus, the axial charge can be used to validate lattice-QCD calculations before studying the momentum dependence of the form factor. In addition, a percent-level first principles calculation of $g_{A}$ could shed light on the neutron lifetime puzzle [13].

Lattice-QCD calculations of baryonic observables are hindered by the well-known exponential growth of the noise relative to the signal, which sets in at large times $[14,15]$. At early times, where the signal-to-noise ratio is favorable, the lattice-QCD correlator data contain significant contributions from several states in an infinite tower. When using a fit to disentangle the higher-lying states from those of interest, some residual, unwanted contamination remains in the parameters of interest. It is imperative, therefore, to demonstrate control over both the noise and the excited-state contamination.

In this work, we use an ensemble generated by the MILC Collaboration [16], which incorporates a sea with equalmass up and down quarks, the strange quark, and the charm quark. MILC uses the highly improved staggered-quark (HISQ) action [17] for the sea quarks; here we use the HISQ action for the valence quarks too. Because staggered fermions have only one component per site and retain a remnant chiral symmetry, they are computationally efficient. Nevertheless, staggered fermions are complicated by the fermion doubling problem, leading to four species, known as tastes, for each fermion field. The four tastes become identical in the continuum limit, leading to an $\mathrm{SU}\left(4 n_{f}\right)$ flavor-taste symmetry for $n_{f}$ flavors. Consequently, the spectrum of staggered lattice baryons is rich and intricate. For nucleons, the spectrum has been classified [18-20], finding many states that have the same properties as the physical nucleon.

In a recent paper, we used staggered baryons to calculate the nucleon mass [20]. Computing nucleon charges is the next step and a necessary one en route to the full momentum dependence of the form factors. As discussed in Ref. [20], it can be advantageous to use unphysical nucleonlike states to carry out the calculation. These states obtain the same properties as the physical nucleon in the continuum limit, where the full $\mathrm{SU}(8)_{\mathrm{FT}}$ flavor(isospin)taste symmetry emerges. For matrix elements such as charges and form factors, however, one must find the correct group-theoretic normalization factors relating nucleonlike matrix elements to their physical counterparts. This exercise is a straightforward if complicated application of the generalization of the Wigner-Eckart theorem to $\mathrm{SU}(8)$.

To demonstrate this approach, we compute the nucleon vector and axial-vector charges on a single MILC HISQ ensemble with lattice spacing $a \approx 0.12 \mathrm{fm}$ and pion mass $M_{\pi} \approx 305 \mathrm{MeV}$. We employ local vector and axial-vector currents. We also outline the steps needed to apply this method to matrix elements of other baryons, with an eye to future studies including staggered baryons, such as $N \rightarrow \Delta$ transition form factors. We find that it is possible, without too much effort, to cope with the challenges that come with staggered valence quarks. As with mesons [21], it is possible to fit the oscillating states but even better to take combinations of adjacent time slices to suppress their contribution before trying such fits. Although multistate Bayesian curve fitting is sufficient, we have code to solve the generalized eigenvalue problem on two-point correlators from our earlier work studying the nucleon mass [20]. Following the suggestion of Ref. [22], we find that a suitably projected three-point function is saturated with the nucleon at reasonable distances. Our research thus points to a third way for mining MILC's library of HISQ ensembles, with its unprecedented scope and very high statistics. Earlier work on these ensembles used a mixed-action setup, with Möbius domain wall [23] or Wilson-clover [24] valence fermions. Such setups entail additional lowenergy constants in the context of chiral perturbation theory and, in the case of Wilson fermions, the danger of exceptional configurations. The remnant chiral symmetry also makes renormalization simpler than with Wilson fermions. For example, here we can obtain the normalization of $g_{A}$ from a vector current.

This paper is organized as follows. In Sec. II, we discuss staggered-baryon correlators, starting with a brief review of the two-point correlator methodology [20]. We then present an overview of our three-point correlators. Here, we also present one of the key results of this paper: the correct normalization of the nucleonlike matrix elements. In Sec. III, we describe strategies for removing excited-state contamination. Section IV provides the details of our simulation, while Sec. V describes Bayesian fits to the correlator data. Our computational results are presented in Sec. VI, including the robustness of our results under variations of our fitting procedure, the renormalization of the bare lattice charge to the physical charges, and the final values for $g_{V}$ and $g_{A}$ on the single ensemble being used. Finally, we compare our results to mixed-action results on the same ensemble and provide our conclusions in Sec. VII. Appendixes A and B present the group theory relating the nucleonlike matrix elements to their physical counterparts, including a numerical demonstration that these derivations are correct.

\section{STAGGERED BARYON CORRELATORS}

For simplicity, we focus here on two flavors, up and down, with isospin symmetry. With staggered fermions, instead of the usual $\mathrm{SU}(2)_{F}$ isospin symmetry, an enlarged $\mathrm{SU}(8)_{\mathrm{FT}}$ flavor-taste symmetry group emerges in the continuum limit. It is important to note that the irreducible flavor-taste representations contain components with nontrivial taste and unexpected isospin. For example, Bailey 
has shown [19] that nucleonlike states exist with unphysical isospin yet masses equal in the continuum limit to the physical tasteless nucleon. In fact, all physics of such nucleonlike states can be related to that of the physical nucleon. In particular, here we show how to relate nucleonlike matrix elements to their physical counterparts. As such, we are allowed to choose any nucleonlike representation, for example, one that reduces the computational complexity.

We use the isospin- $-\frac{3}{2}$ operators that transform in the 16 irrep of the geometric time slice group (GTS) [18,25], as presented in Ref. [20]. They are less complicated to analyze because only a single nucleonlike taste appears in the spectrum. On the other hand, this irrep contains contributions from three $\Delta$-like tastes.

\section{A. Two-point correlators}

Using the same notation as in Ref. [20], the two-point correlators read

$$
C_{2 \mathrm{pt}}^{\left(r_{1}, r_{2}\right)}=\frac{1}{16} \sum_{s, \vec{D}} \sum_{\vec{x}}\left\langle B_{s \vec{D}}^{\left(r_{2}\right)}(\vec{x}, t) \bar{B}_{s \vec{D}}^{\left(r_{1}\right)}(0)\right\rangle,
$$

using sink and source operators $B_{s \vec{D}}^{\left(r_{2}\right)}(\vec{x}, t)$ and $\bar{B}_{s \vec{D}}^{\left(r_{1}\right)}(0)$ defined in Ref. [20]. To increase the statistical precision, we average over the eigenvalues $s= \pm$ of the staggered rotation in the $x-y$ plane, and also the eight corners of the cube $\vec{D}$; together, $s$ and $\vec{D}$ label the components of the 16 irrep. Here, $r_{1}, r_{2}=2,3,4,6$ represent four different operator constructions, or "classes" [18-20], as well as other possible properties, such as smearing.

\section{B. Staggered-baryon matrix elements}

In this work, we are specifically interested in the isovector nucleon vector and axial-vector charges, namely $g_{V}$ and $g_{A}$, respectively. These are defined through the nucleon matrix elements

$$
\left\langle N\left|\left(\bar{u} \Gamma_{J} u-\bar{d} \Gamma_{J} d\right)\right| N\right\rangle=g_{J} \bar{u}_{N} \Gamma_{J} u_{N},
$$

where $\Gamma_{A}=\gamma_{z} \gamma_{5}$ or $\Gamma_{V}=\gamma_{4}, u$ and $d$ are continuum-QCD up- and down-quark fields, and $u_{N}$ is the nucleon spinor at zero momentum.

We calculate these nucleon matrix elements using (highly improved) staggered quarks. To achieve this, we must extend the mass relations of Bailey [19] to matrix elements. The baryonlike matrix elements and the physical matrix elements are related through symmetry transformations in the continuum. In the Appendixes, we find the appropriate Clebsch-Gordan coefficients that relate the single-taste baryon matrix elements to the physical tasteless QCD matrix elements by applying the generalized WignerEckart theorem of $\mathrm{SU}(8)_{\mathrm{FT}}$.

The correctly normalized three-point correlators for our baryonlike operators are then

$$
\begin{aligned}
& C_{V}^{\left(r_{1}, r_{2}\right)}(t, \tau) \\
& =-\frac{1}{16} \sum_{\vec{D}} \sum_{\vec{x}, \vec{y}} \mathcal{S}_{V}(\vec{D})\left(\left\langle B_{-\vec{D}}^{\left(r_{2}\right)}(\vec{x}, t) V(\vec{y}, \tau) \bar{B}_{-\vec{D}}^{\left(r_{1}\right)}(0)\right\rangle\right. \\
& \left.\quad+\left\langle B_{+\vec{D}}^{\left(r_{2}\right)}(\vec{x}, t) V(\vec{y}, \tau) \bar{B}_{+\vec{D}}^{\left(r_{1}\right)}(0)\right\rangle\right), \\
& C_{A}^{\left(r_{1}, r_{2}\right)}(t, \tau) \\
& =\frac{1}{16} \sum_{\vec{D}} \sum_{\vec{x}, \vec{y}} \mathcal{S}_{A}(\vec{D})\left(\left\langle B_{-\vec{D}}^{\left(r_{2}\right)}(\vec{x}, t) A(\vec{y}, \tau) \bar{B}_{-\vec{D}}^{\left(r_{1}\right)}(0)\right\rangle\right. \\
& \left.\quad-3\left\langle B_{+\vec{D}}^{\left(r_{2}\right)}(\vec{x}, t) A(\vec{y}, \tau) \bar{B}_{+\vec{D}}^{\left(r_{1}\right)}(0)\right\rangle\right),
\end{aligned}
$$

where $t$ is the source-sink separation time and $\tau$ is the current insertion time. The factor -1 in front of $C_{V}$ and the factor -3 in front of the second term of $C_{A}$ come from the group theory just described. Without these factors, these correlators would not yield the desired nucleon charges. For baryon operators and currents in other GTS irreps, different prefactors arise. In Eqs. (2.3) and (2.4), we sum over unit-cube sites $\vec{D}$ with weights $\mathcal{S}_{J}(\vec{D})(J=V, A)$ and have a separate term for each value of $s= \pm 1$.

Equations (2.3) and (2.4) introduce local currents

$$
\begin{aligned}
V(\vec{y}, \tau) & =\mathcal{S}_{V}(\vec{y})\left(\bar{\chi}_{u}(\vec{y}, \tau) \chi_{u}(\vec{y}, \tau)-\bar{\chi}_{d}(\vec{y}, \tau) \chi_{d}(\vec{y}, \tau)\right), \\
\mathcal{S}_{V}(\vec{A}) & =(-1)^{\left(A_{x}+A_{y}+A_{z}\right) / a} \\
A(\vec{y}, \tau) & =\mathcal{S}_{A}(\vec{y})\left(\bar{\chi}_{u}(\vec{y}, \tau) \chi_{u}(\vec{y}, \tau)-\bar{\chi}_{d}(\vec{y}, \tau) \chi_{d}(\vec{y}, \tau)\right), \\
\mathcal{S}_{A}(\vec{A}) & =(-1)^{A_{z} / a}
\end{aligned}
$$

where $\chi_{f}$ is the field in the HISQ action of flavor $f$. The local vector and axial-vector currents, $V$ and $A$, have spin taste $\gamma_{4} \otimes \xi_{4}$ and $\gamma_{z} \gamma_{5} \otimes \xi_{z} \xi_{5}$ [25], respectively. The opposite-parity partners then arise from spin taste $\gamma_{5} \otimes \xi_{5}$ and $\gamma_{z} \gamma_{4} \otimes \xi_{z} \xi_{4}$, respectively. Because these local currents are not derived from Noether's theorem, they require a finite renormalization, that is, $Z_{V} V$ and $Z_{A} A$ have the same matrix elements as the continuum isovector currents in Eq. (2.2).

In the limit $\tau \rightarrow \infty$ and $t-\tau \rightarrow \infty$, the ratio of the threepoint to the two-point correlators approaches the desired nucleon charge

$$
\frac{C_{g_{J}}^{\left(r_{1}, r_{2}\right)}(t, \tau)}{C_{2 \mathrm{pt}}^{\left(r_{1}, r_{2}\right)}(t)} \underset{t \rightarrow \tau \rightarrow \infty}{\stackrel{\tau \rightarrow \infty}{\longrightarrow}} \tilde{g}_{J}
$$

where $\tilde{g}_{J}$ is now the bare lattice charge, that is, $g_{J}=Z_{J} \tilde{g}_{J}$. In practice, of course, we compute the correlators for several values of $t$ and $\tau$ and fit the $t$ and $\tau$ dependence to extract the charges.

The finite renormalization factors $Z_{J}$ are determined first by noting that the remnant chiral symmetry requires 
$Z_{A}=Z_{V}+\mathrm{O}\left(m_{q} a\right)^{2}$. At zero momentum transfer, the vector current simply counts the number of up quarks minus the number of down quarks, for the proton $2-1=1$. One could, thus, define $Z_{V}$ by demanding $Z_{V} \tilde{g}_{V}=g_{V}=1$. Here, however, we prefer to define $Z_{V}$ via a similar relation obtained from a pseudoscalar-meson matrix element [26], then use the result to renormalize our nucleon matrix elements. With this choice our result for $g_{V}$ is a genuine test of our methodology.

\section{EXCITED-STATE CONTAMINATION}

Excited-state contamination is one of the most difficult challenges when accurately estimating nucleon matrix elements from lattice QCD. The problem is even more complicated with staggered nucleons because of the presence of negative-parity and low-lying $\Delta$-like states in the spectrum, which nonstaggered formulations do not contain. These are both significant sources of excited-state contamination in the present calculation. We have, however, demonstrated control of excited-state contamination when extracting nucleon physics from two-point staggeredbaryon correlators [20]. Here we describe extensions of those techniques to the three-point correlators of the present work. In particular, we show how to suppress contributions from the lowest-lying negative-parity states and the lowest three $\Delta$-like tastes.

\section{A. Negative-parity states}

Let $C_{2 \mathrm{pt}}(t)$ and $C_{3 \mathrm{pt}}(t, \tau)$ be any staggered-baryon correlators. The source-sink separation is denoted $t$ and the current insertion time is denoted $\tau$. Any staggered operator that is local in time will create negative parity states, which in turn causes the characteristic oscillations in time. This is obvious from the correlators spectral decomposition

$$
\begin{aligned}
C_{2 \mathrm{pt}}(t)=z_{+} & \bar{z}_{+} e^{-M_{+} t}+(-1)^{t / a} z_{-} \bar{z}_{-} e^{-M_{-} t}+\cdots, \\
C_{3 \mathrm{pt}}(t, \tau)= & z_{+} A_{++} \bar{z}_{+} e^{-M_{+} t}+(-1)^{t / a} z_{-} A_{--} \bar{z}_{-} e^{-M_{-} t} \\
& +(-1)^{(t-\tau) / a_{+}} z_{+} A_{+-} \bar{z}_{-} e^{-M_{+} \tau} e^{-M_{-}(t-\tau)} \\
& +(-1)^{\tau / a} z_{-} A_{-+} \bar{z}_{+} e^{-M_{-} \tau} e^{-M_{+}(t-\tau)} \\
& +\cdots,
\end{aligned}
$$

where $M_{ \pm}$are the lowest-lying \pm parity masses, $\bar{z}_{ \pm}$and $z_{ \pm}$ are, respectively, the source and sink overlap factors for states of parity \pm , and $A_{ \pm \pm}$and $A_{ \pm \mp}$ are the transition matrix elements. For simplicity, we have ignored backward propagating terms proportional to $e^{-M_{ \pm}(T-t)}$, which are assumed to contribute negligibly in the following.

Equation (3.2) shows that the terms involving negative parity states change sign when either $t / a$ or $\tau / a$ change by one unit. With this in mind, a time-averaging procedure can be applied to suppress the negative parity contributions to the correlator. A similar scheme was deployed in Ref. [21]. The first ingredient is

$$
\begin{array}{r}
C_{2 \mathrm{pt}}^{\prime}(t)=e^{-a M_{\mathrm{snk}}} C_{2 \mathrm{pt}}(t)+C_{2 \mathrm{pt}}(t+a), \\
C_{3 \mathrm{pt}}^{\prime}(t, \tau)=e^{-a M_{\mathrm{snk}}} C_{3 \mathrm{pt}}(t, \tau)+C_{3 \mathrm{pt}}(t+a, \tau),
\end{array}
$$

where we call $a M_{\text {snk }}$ the time-averaging parameter. Substituting this expression into the spectral decomposition in Eq. (3.2), one sees that the functional forms of primed correlators are unchanged except that the sink overlap factors become

$$
\begin{gathered}
z_{+} \rightarrow z_{+}\left(e^{-a M_{\mathrm{snk}}}+e^{-a M_{+}}\right), \\
z_{-} \rightarrow z_{-}\left(e^{-a M_{\mathrm{snk}}}-e^{-a M_{-}}\right) .
\end{gathered}
$$

If one chooses $a M_{\text {snk }}=a M_{-}$, then terms with the $M_{-}$state at the sink will vanish, while the overlap factors for the positive parity states become slightly larger. In practice, the time-averaging parameter does not need to be exact to suppress the negative-parity states.

A similar time-averaging parameter, $a M_{\text {src }}$, can be introduced to reduce the negative parity contributions at the source via

$$
\begin{array}{r}
C_{2 \mathrm{pt}}^{\prime \prime}(t)=e^{-a M_{\mathrm{src}}} C_{2 \mathrm{pt}}(t)+C_{2 \mathrm{pt}}(t+a), \\
C_{3 \mathrm{pt}}^{\prime \prime}(t, \tau)=e^{-a M_{\mathrm{src}}} C_{3 \mathrm{pt}}(t, \tau)+C_{3 \mathrm{pt}}(t+a, \tau+a) .
\end{array}
$$

Again, this step does not alter the functional forms of the two- and three-point correlators but replaces the source overlap factors by

$$
\begin{gathered}
\bar{z}_{+} \rightarrow \bar{z}_{+}\left(e^{-a M_{\mathrm{src}}}+e^{-a M_{+}}\right), \\
\bar{z}_{-} \rightarrow \bar{z}_{-}\left(e^{-a M_{\mathrm{src}}}-e^{-a M_{-}}\right) .
\end{gathered}
$$

If several negative parity states contribute significantly to the data, successive applications of this procedure, with suitable parameters $\left[a M_{\mathrm{src}}^{(1)}, a M_{\mathrm{src}}^{(2)}, \ldots\right]$ and $\left[a M_{\mathrm{snk}}^{(1)}\right.$, $\left.a M_{\mathrm{snk}}^{(2)}, \ldots\right]$, can appreciably suppress them. On the other hand, because the relative error in the correlators becomes larger with time, too much time averaging renders the data statistically less precise. Moreover, time-averaging reduces the available $\tau$ range in the modified correlators, thereby producing fewer data for the fit. For each dataset, some study is necessary to strike an optimal balance.

\section{B. $\Delta$-like states}

Another source of excited-state contamination arises from the presence of the three $\Delta$-like states in the 16-irrep correlators. With four different classes of interpolators at both the source and the sink, we adopt the strategy from 
Ref. [22] and solve the generalized eigenvalue problem (GEVP) [27]. In Ref. [20], we applied the GEVP to our two-point correlators and successfully disentangled the nucleonlike state from the $\Delta$-like states. We extend that strategy to the three-point functions here.

Given a matrix two-point correlator, $\mathbf{C}_{2 \mathrm{pt}}(t)$, the left and right nucleon eigenvectors, $\boldsymbol{u}\left(t_{1}, t_{0}\right)$ and $\boldsymbol{v}\left(t_{1}, t_{0}\right)$, are the solutions of

$$
\begin{aligned}
\mathbf{C}_{2 \mathrm{pt}}\left(t_{1}\right) \boldsymbol{u}\left(t_{1}, t_{0}\right) & =\lambda\left(t_{1}, t_{0}\right) \mathbf{C}_{2 \mathrm{pt}}\left(t_{0}\right) \boldsymbol{u}\left(t_{1}, t_{0}\right), \\
\boldsymbol{v}\left(t_{1}, t_{0}\right) \mathbf{C}_{2 \mathrm{pt}}\left(t_{1}\right) & =\lambda\left(t_{1}, t_{0}\right) \boldsymbol{v}\left(t_{1}, t_{0}\right) \mathbf{C}_{2 \mathrm{pt}}\left(t_{0}\right) .
\end{aligned}
$$

Here, we focus on the eigenvectors for the nucleonlike state, the ones with the lowest eigenvalues, and put the others aside. These eigenvectors optimize the projection onto the nucleonlike state in both the two- and the threepoint correlators via

$$
\begin{aligned}
C_{2 \mathrm{pt}}(t) & =\boldsymbol{v}\left(t_{1}, t_{0}\right) \mathbf{C}_{2 \mathrm{pt}}(t) \boldsymbol{u}\left(t_{1}, t_{0}\right), \\
C_{3 \mathrm{pt}}(t, \tau) & =\boldsymbol{v}\left(t_{1}, t_{0}\right) \mathbf{C}_{3 \mathrm{pt}}(t, \tau) \boldsymbol{u}\left(t_{1}, t_{0}\right) .
\end{aligned}
$$

One has to decide which $t_{1}$ and $t_{0}$ to use in Eqs. (3.11) and (3.12). The stability of our results under such variations will be discussed in Sec. IV. Below we call the correlators in Eqs. (3.13) and (3.14) the nucleon-optimized two- and three-point correlators.

To summarize our strategy, we start with the correlators in Eqs. (2.1), (2.4), and (2.3), and apply two iterations of time averaging at both the source and the sink, and then project the time-averaged correlation matrix as in Eq. (3.14). The time averaging suppresses the negative parity states contributions, and the projection suppresses the $\Delta$-like baryons contributions.

\section{SIMULATION DETAILS}

To demonstrate the feasibility of nucleon matrix elements with staggered quarks, we use a single gauge ensemble, which was generated by the MILC Collaboration [16]. MILC implemented the one-loop, tadpole-improved Lüscher-Weisz gauge action [28], as well as the HISQ action [17] for the sea, which contains equal-mass up and down quarks, the strange quark, and the charm quark. In this work, we also employ the HISQ action for the valence quarks, with the same mass as the up-down sea quarks.

The ensemble has dimension $L^{3} \times T=24^{3} \times 64$, a lattice spacing $a=0.1222(3) \mathrm{fm}$ (determined from the $F_{p 4 s}$ massindependent scheme [29]), a pion mass $M_{\pi} \approx 305 \mathrm{MeV}$, and a light-to-strange-quark mass ratio of $1 / 5$. Other parameters of this ensemble are listed in Ref. [29]. Note that the CalLat [23] and the PNDME [24] Collaborations have both used this same ensemble to calculate $g_{A}$, albeit with either the Möbius domain wall or Wilson-clover valence fermion actions, respectively.
We generate the two- and three-point correlators according to Eqs. (2.1), (2.3), and (2.4). We measure each correlator on 872 configurations, and further increase the statistics by randomly placing the corner-wall sources on eight maximally separated time slices to give a total of 6976 measurements per correlator. (The $\tau / a=7$ correlators have only four time sources per configuration.)

We block all measurements in a single gauge configuration and every four consecutive gauge trajectories to avoid autocorrelations. The covariance matrix between different correlator components are estimated with the nonlinear shrinkage method [30] to avoid ill-conditioning from finite sample sizes.

As described in Ref. [20], we use corner-wall sources to optimize the signal-to-noise ratio and point sinks. In the present work, we remove the Coulomb-gauge fixed links, as we have empirically observed that leaving out the links has little effect on correlators but with the added advantage of a simpler code. Here we also incorporate the Wuppertal smearing [31,32] at the sink by applying

$$
\chi^{(n)}=\left(1+\frac{3 \sigma^{2}}{32 a^{2} N} \Delta\right) \chi^{(n-1)},
$$

$\Delta \chi(\vec{x})=-6 \chi(\vec{x})+\sum_{i=1}^{3}\left[\chi\left(x_{i}+2 a\right)+\chi\left(x_{i}-2 a\right)\right]$

in order to reduce excited-state contamination. In Eq. (4.1), $n$ is the $n$th iteration of $N$ total iterations; all shifts are stride 2 to preserve the staggered symmetries. We include the appropriate gauge transporters to make the smearing gauge covariant [20], but for succinctness they are omitted from Eq. (4.2). We generate data with two different root-meansquared (rms) smearing radii, $\sigma$, which are about 0.2 and $0.6 \mathrm{fm}$. We label the two smearings as Gr2.0N30 and Gr6.0N70.

For the three-point correlators, we invert the propagators from the current insertion to obtain all operator classes at the sink. Five current insertion times, $\tau / a=[3,4,5,6,7]$, are generated for both the vector and the axial-vector current insertions.

On each configuration, we solve the staggered Dirac equation

$$
N_{\text {sol }}^{\text {2pt }}=N_{\mathrm{c}} N_{\vec{D}} N_{\text {src }}
$$

times to construct two-point functions, and

$$
N_{\mathrm{sol}, \tau}^{3 \mathrm{pt}}=N_{\mathrm{c}} N_{\vec{D}} N_{J} N_{\mathrm{src}, \tau},
$$

more times for each three-point function current insertion time $\tau$. The total number of solves computed per configuration is then 


$$
N_{\mathrm{sol}}=N_{\mathrm{sol}}^{2 \mathrm{pt}}+\sum_{\tau=3}^{7} N_{\mathrm{sol}, \tau}^{3 \mathrm{pt}}
$$

$N_{\mathrm{c}}$ is the number of colors, $N_{\vec{D}}$ the number of corners in the spatial cube (analogous to the four Dirac components of Wilson or domain-wall fermions), $N_{\text {src }}$ the number of two-point function time sources, $N_{\mathrm{src}, \tau}$ the number of three-point function time sources for current insertion time $\tau$, and $N_{J}$ the number of unique currents. In this work, $N_{\mathrm{c}}=3, N_{\vec{D}}=8, N_{J}=2$ for the vector and axial-vector currents, and $N_{\mathrm{src}}=8$. For $\tau / a=7, N_{\mathrm{src}, \tau}=4$, while for the other source-current separations $N_{\mathrm{src}, \tau}=8$. The total is $N_{\text {sol }}=1920$. The Wuppertal smearing is applied at the sink so it requires no extra solves. The four operators that enable the GEVP analysis also do not require extra solves if $N_{\vec{D}}=8$; at the expense of the operator basis, which reduces excited-state contaminations, $N_{\vec{D}}$ could be chosen as small as 3.

To eliminate the unwanted negative parity states, we then pass all the correlators through two iterations of time averaging using Eqs. (3.4) and (3.7) with

$$
\left[a M_{\mathrm{src}}^{(1)}, a M_{\mathrm{src}}^{(2)}\right]=\left[a M_{\mathrm{snk}}^{(1)}, a M_{\mathrm{snk}}^{(2)}\right]=[0.9,1.1] .
$$

These two numbers are based on an observation in Ref. [20] that the lowest-lying negative parity state seems to have energy around the $S$-wave $N \pi$ state, which in this ensemble is about 0.9 in lattice units. We then set the second averaging parameters about $a M_{\pi} \sim 0.2$ higher than the first ones, which again is consistent with our findings in Ref. [20]. As the goal is to suppress the negative-parity states, the accuracy of these parameters is not crucial. Note that each iteration of the source time averaging in Eq. (3.8) reduces the current insertion time slices by one, so the timeaveraged three-point correlators have only $\tau / a=[3,4,5]$. The smearing in Eq. (3.4), on the other hand, does not reduce the range for $\tau / a$.

After time averaging, we solve for the left and right eigenvectors using Eq. (3.12) in order to optimize our correlators as in Eq. (3.14). To ensure the robustness of our fitting methodology, we test the stability of our results under variations of the choice of $t_{0}$. To do so, we compute the effective mass of the optimized two-point correlators, which we define as

$$
a M_{\mathrm{eff}}(t) \equiv \frac{1}{2} \ln \left(\frac{C_{2 \mathrm{pt}}(t)}{C_{2 \mathrm{pt}}(t+2 a)}\right)
$$

The $t_{0}$ stability plots are shown in Fig. 1. Because the statistical errors of the eigenvectors are taken into account by solving Eqs. (3.11) and (3.12) via bootstrap resampling, the errors of the left and right eigenvectors, and hence, the optimized two-point correlators grow as $t_{0} / a$ increases. However, because of the strong correlation between $C_{2 \mathrm{pt}}(t)$
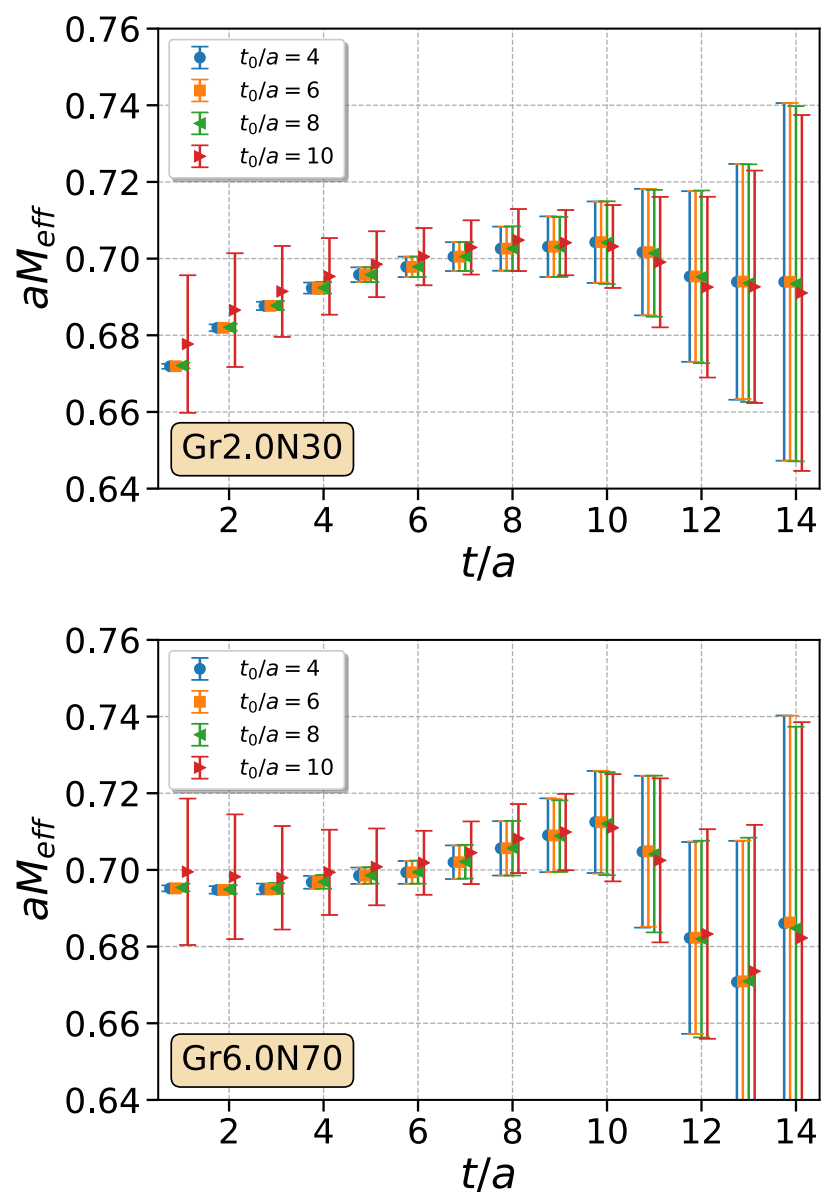

FIG. 1. Effective masses of the nucleon-optimized correlators as a function of the source-sink separation time $t$. The top plot has Wuppertal sink smearing radius $\sigma_{\text {rms }}=0.2 \mathrm{fm}(\mathrm{Gr} 2.0 \mathrm{~N} 30)$, and the bottom $\sigma_{\mathrm{rms}}=0.6 \mathrm{fm}$ (Gr6.0N70). The time-averaging parameters are given in Eq. (4.6). Different colored points represent different choices of $t_{0}$ when solving the GEVP equation in Eq. (3.12), as shown in the legends, and are offset slightly for clarity.

and $C_{2 \mathrm{pt}}(t+2 a)$, the effective masses are insensitive to the errors of eigenvectors. This is why the errors stay relatively constant for $t_{0} / a=4,6$, and 8 ; for $t_{0} / a=10$, on the other hand, the eigenvectors' errors become so large that they dominate the errors at small $t / a$, but again become insignificant as $t$ increases. We observe that $t_{0} / a=4,6$, and 8 all produce similar results, so we choose $t_{0} / a=6$ for the subsequent analysis. Similarly, we vary $\left(t_{1}-t_{0}\right) / a$ from 2 to 6 and find, again, that the differences are negligible, so we fix $\left(t_{1}-t_{0}\right) / a=2$.

Since we have normalized the nucleonlike three-point correlators correctly, and each of the correlator transformations that we perform preserve the functional form of the spectral decomposition, the optimized three-to-two-point correlator ratios converge to the desired nucleon charges in the large-time limits. In Figs. 2 and 3, we plot the ratio of the nucleon-optimized three-to-two-point correlators, with and without time averaging at the source and sink. The left 

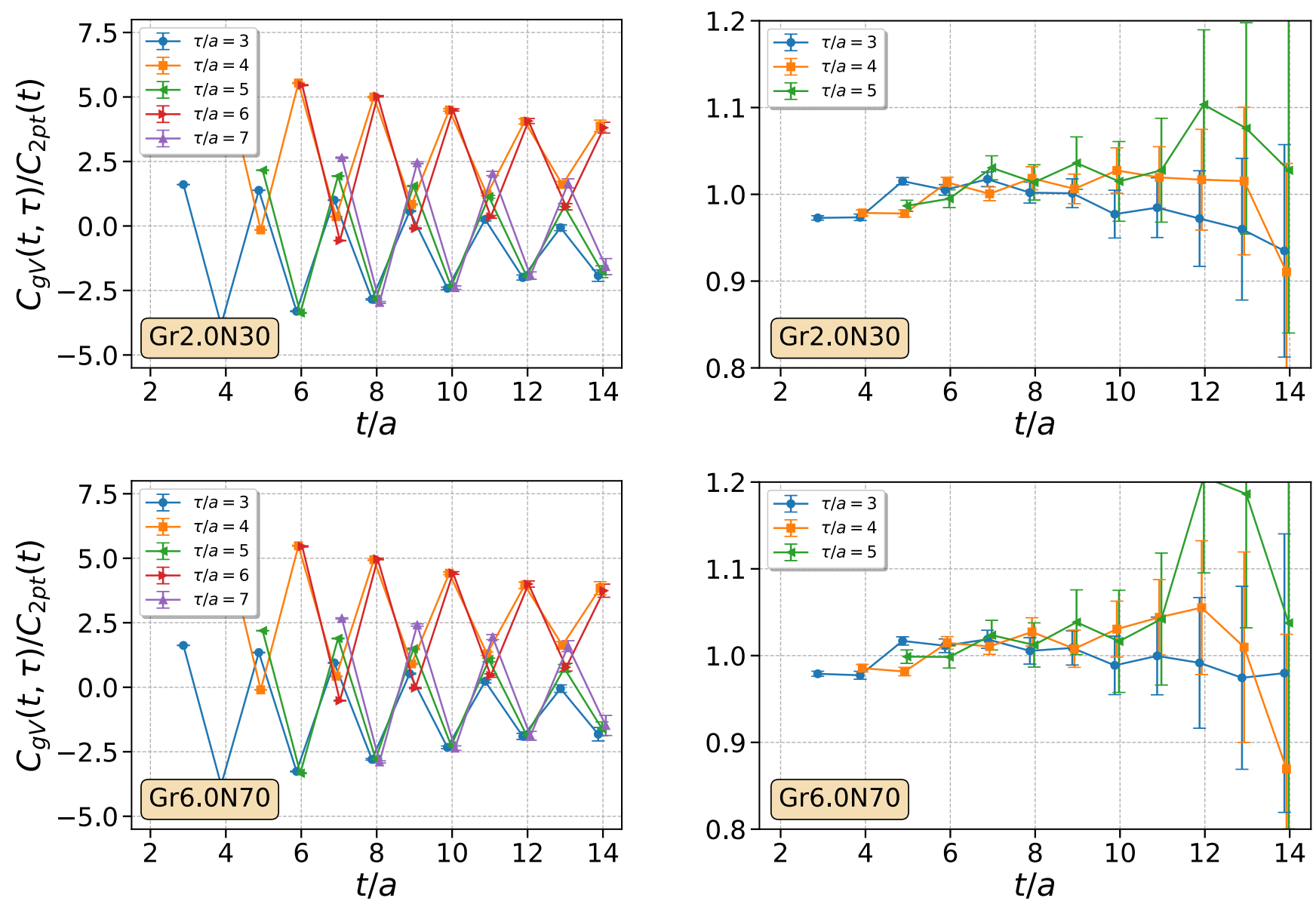

FIG. 2. The $g_{V}$ three-point to two-point nucleon-optimized correlator ratio as a function of the source-sink separation time $t$, and current insertion time $\tau$. Correlators are labeled by the rms Wuppertal smearing radii, $\sigma_{\text {rms }}=0.2 \mathrm{fm}(\mathrm{Gr} 2.0 \mathrm{~N} 30)$ and $\sigma_{\text {rms }}=0.6 \mathrm{fm}$ (Gr6.0N70). In the limits $\tau, t-\tau \rightarrow \infty$, this ratio converges to the bare $g_{V}$ nucleon charge. The correlators in the left column are not time averaged with the oscillation suppressing procedure described in Sec. III. The right column shows data that are time-averaged with the parameters given in Eq. (4.6), yielding much smoother curves reminiscent of nonstaggered fermion correlators.

column shows the optimized correlators without time averaging, and the right column shows them time averaged with the parameters given in Eq. (4.6). The two smearings are shown in the top (Gr2.0N30) and bottom (Gr6.0N70) rows. Significant oscillations are clearly present in the unaveraged correlators, particularly for the vector current in Fig. 2. This is expected, because the parity partner of the vector current is the pseudoscalar current $P$, and the $\langle N \pi|P| N\rangle$ matrix element gives a large contribution to the vector-current data and, thus, causes large oscillations. For the $g_{A}$ data, on the other hand, the parity partner of the axial current is the tensor current $T_{z 4}$, and when the nucleon is at rest $\left\langle N\left|T_{z 4}\right| N\right\rangle=0$. Consequently, the first nonzero contribution in the axial-vector parity partner channel will likely be from $\left\langle N \pi\left|T_{z 4}\right| N\right\rangle$, leaving small oscillations.

\section{CORRELATOR FITTING}

We apply the Bayesian fitting methodology implemented in CORRFITTER [33] to extract the nucleon mass and matrix elements. We observe in Fig. 2 that the vector correlators have noticeable oscillatory contributions, whereas the axial-vector correlators shown in Fig. 3 do not. Further, the vector correlators seem relatively insensitive to our choice of Wuppertal smearing. We perform separate fits to the vector and axial-vector correlators, but include their correlations through bootstrapping.

It should be stressed that, after applying the excited-state suppression techniques from Sec. III, the interpretation of the higher exponentials in the correlators is ambiguous. For the positive-parity channel, the first "excited state" could be a mixture of any leftover $\Delta$-like states, the $P$-wave $N \pi$ states, or other finite volume energy levels higher up in the spectrum that are related to resonances. For the negativeparity channel, we found in Ref. [20] that the ground state is likely to contain $S$-wave $N \pi$ states. The time-averaging procedure to cancel out the negative-parity states makes identification of these states even more ambiguous. Regardless of the origin, we can treat the excited states as nuisance parameters and fit them away with an 

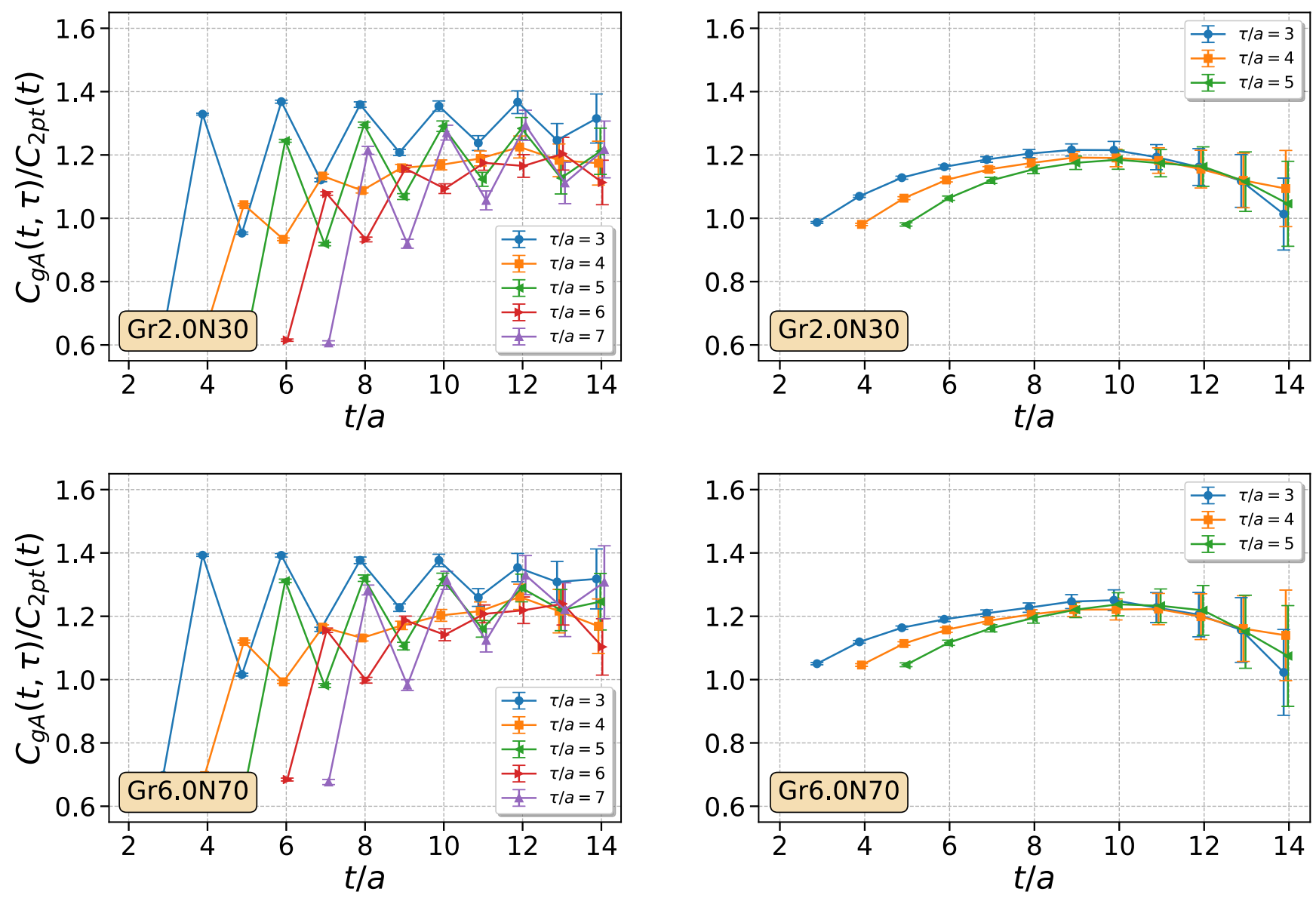

FIG. 3. Identical to Fig. 2 but with $g_{A}$ instead of $g_{V}$. See the caption of Fig. 2 for further details.

exponential fit function. In this case, each excited exponential mass parameter describes a conglomeration of several eigenstates of the Hamiltonian. Still, we will refer to each exponential in the fit function as a state without necessarily identifying it with any single eigenstate. As discussed in Ref. [20], the stability of the extracted fit parameters as a function $t_{\min }$ indicates a lack of excitedstate contamination, as long as they are modeled accurately. This $t_{\min }$ stability plot is shown in Fig. 5 and is discussed in Sec. V B.

\section{A. Functional forms for fitting}

For the $g_{A}$ analysis, we perform simultaneous fits to the optimized two- and three-point correlators, and include both the Gr2.0N30 and the Gr6.0N70 sink smearings. Observation of the strong suppression of excited states in Fig. 2 leads us to use a fit ansatz that contains two positiveparity states and one negative-parity state:

$$
\begin{aligned}
C_{2 \mathrm{pt}}^{\sigma, \mathrm{fit}}(t)= & z_{+0}^{\sigma} \bar{z}_{+0} e^{-M_{+0} t}+z_{+1}^{\sigma} \bar{z}_{+1} e^{-M_{+1} t} \\
& +(-1)^{t / a} z_{-0}^{\sigma} \bar{z}_{-0} e^{-M_{-0} t}
\end{aligned}
$$

$$
\begin{aligned}
C_{A}^{\sigma, \mathrm{fit}}(t, \tau)= & \sum_{i, j=0}^{1} z_{+i}^{\sigma} A_{+i,+j} \bar{z}_{+j} e^{-M_{+i} \tau} e^{-M_{+j}(t-\tau)} \\
& +z_{-0}^{\sigma} A_{-0,-0} \bar{z}_{-0 i}(-1)^{t / a} e^{-M_{-0} t} \\
& +\sum_{i=0}^{1} z_{-0}^{\sigma} A_{-0,+i} \bar{z}_{+i}(-1)^{(t-\tau) / a} e^{-M_{+i} \tau} e^{-M_{-0}(t-\tau)} \\
& +\sum_{i=0}^{1} z_{+i}^{\sigma} A_{+i,-0} \bar{z}_{-0}(-1)^{\tau / a} e^{-M_{-0} \tau} e^{-M_{+i}(t-\tau)}
\end{aligned}
$$

Here, $M_{+0}=M_{N}$ is the nucleon mass, $M_{+1}$ is the mass of the first residual positive-parity excited state, $\bar{z}_{+i}$ and $z_{+i}^{\sigma}$ are their source and sink overlap factors (with sink smearing $\sigma=0.2,0.6 \mathrm{fm}), M_{-0}$ is the mass of the residual negative-parity state, and $\bar{z}_{-0}$ and $z_{-0}^{\sigma}$ are the source and sink overlap factors. The terms $A_{ \pm i, \pm j}$ are the unrenormalized axial-vector matrix elements, with $A_{+0,+0}=\tilde{g}_{A}$ the desired bare axial charge. Note that the two-point correlator terms involving the finite temporal $T$ extent are not included here since we average our data symmetrically around the $T / 2$ point as described in Ref. [20]. 
In the Bayesian fit, we choose Gaussian priors for the ground-state masses, overlap factors, and matrix elements. We choose log-normal priors for the mass differences between adjacent states to enforce the ordering of states. It has been observed empirically that the nucleon mass has an approximate linear dependence on the pion mass (see, for example, Ref. [34]), so we choose a prior of $1100 \pm$ $200 \mathrm{MeV}$ for the nucleon mass on our ensemble with $M_{\pi}=305 \mathrm{MeV}$. We put a wide prior of $300 \pm 300 \mathrm{MeV}$ centered at the $\Delta$-like mass for the mass splitting $M_{+1}-$ $M_{+0}$ to accommodate for potential mixing of many physical states. For the same reason, we also impose a wide mass prior of $1600 \pm 500 \mathrm{MeV}$ for the negativeparity mass $M_{-0}$ centered at the $S$-wave $N \pi$ state. All prior choices are summarized in Table I.

A priori, we have no knowledge of the sign or magnitude of the overlap factors. Consequently, all overlap factors are effectively unconstrained. In practice, we have normalized the correlators such that their values are much less than 1 for all $t$, so the overlap priors are chosen to be $0 \pm 5$ for all states. Very wide priors of $0 \pm 5$ are chosen for all matrix elements, apart from $\tilde{g}_{A}=A_{+0,+0}$, for which we choose a wide prior of $1.2 \pm 0.3$ centered near the PDG [12] value of $g_{A}$. As discussed below in Sec. VI, we know from other work with pseudoscalar mesons that $Z_{A}$ is close enough to unity not to influence the choice of prior.

For the $g_{V}$ analysis, we use the same two-point functional form as Eq. (5.1). However, for the three-point correlators we use

$$
\begin{aligned}
C_{g_{V}}^{\sigma, \mathrm{fit}}(t, \tau)= & \sum_{i=0}^{1} z_{+i}^{\sigma} V_{+i,+i} \bar{z}_{+i} e^{-M_{+i} t} \\
& +z_{-0}^{\sigma} V_{-0,-0} \bar{z}_{-0 i}(-1)^{t / a} e^{-M_{-0} t} \\
& +\sum_{i=0}^{1} z_{-0}^{\sigma} V_{-0,+i} \bar{z}_{+i}(-1)^{(t-\tau) / a} e^{-M_{+i} \tau} e^{-M_{-0}(t-\tau)} \\
& +\sum_{i=0}^{1} z_{+i}^{\sigma} V_{+i,-0} \bar{z}_{-0}(-1)^{\tau / a} e^{-M_{-0} \tau} e^{-M_{+i}(t-\tau)}
\end{aligned}
$$

TABLE I. Summary of the prior choices for the fit Ansätze given in Eqs. (5.1), (5.2), and (5.3). All prior distributions are Gaussian, except for $M_{+1}-M_{+0}$, which is log-normal.

\begin{tabular}{lc}
\hline \hline Quantity & Prior value \pm width \\
\hline$M_{+0}=$ nucleon mass & $1100 \pm 200 \mathrm{MeV}$ \\
$M_{+1}-M_{+0}$ & $300 \pm 300 \mathrm{MeV}$ \\
$M_{-0}$ & $1600 \pm 500 \mathrm{MeV}$ \\
$A_{+0,+0}=\tilde{g}_{A}$ & $1.2 \pm 0.3$ \\
$V_{+0,+0}=\tilde{g}_{V}$ & $1.0 \pm 0.3$ \\
$A_{i, j} ; i \neq+0, j \neq+0$ & $0.0 \pm 5.0$ \\
$V_{i, j} ; i \neq+0, j \neq+0$ & $0.0 \pm 5.0$ \\
\hline \hline
\end{tabular}

where the notation is identical to that of Eq. (5.2). The $V_{i, j}$ are the unrenormalized vector matrix elements. The $V_{+i,+j}$ with $i \neq j$ are omitted on the first line of Eq. (5.3), because they are forbidden by vector charge conservation, up to small discretization effects. The priors are also identical to the $g_{A}$ fits except for the bare vector charge, $\tilde{g}_{V}$. Given that the renormalization constant is close to unity, we choose the $\tilde{g}_{V}$ prior to be $1.0 \pm 0.3$.

\section{B. Fit stability}

The most important part of the nucleon matrix element fitting procedure is separating the nucleon observables of interest from the excited-state contributions. To demonstrate the lack of excited-state contamination, we examine the stability of the observables as choices in the fit are varied. Specifically, we vary $t_{\min }, \Delta \tau_{\min }$, and $t_{\max }$ where $t_{\min }$ is the minimum source-sink separation time that we include in our two-point correlator fits, $\Delta \tau_{\min }$ is the minimum source-sink separation time after the current insertion time, $\tau$, that we include in our three-point correlator fits, and $t_{\max }$ is the maximum source-sink separation time. The nominal parameters for the nominal fits are given in Table II.

We plot the stability of the extracted $M_{N}\left(\tilde{g}_{V}\right.$ and $\left.\tilde{g}_{A}\right)$ as a function of $t_{\min }$ and $\Delta \tau_{\min }$ in Fig. 4 (Fig. 5). The $x$ axes are different choices of $t_{\min }$, and the $y$ axes are the corresponding observables. The four different choices of $\Delta \tau_{\min }$ are also shown slightly displaced for each $t_{\min }$. The solid squares are the nominal fits with parameters given in Table II. As can be seen in Fig. 4, the extracted nucleon mass is stable as a function of $t_{\min } / a$ and $\Delta \tau_{\min } / a$, which illustrates the lack of excited-state contamination in these posteriors. Similar behavior is seen for $g_{V}$ in Fig. 5. The only noticeable structure in the stability plots is for $g_{A}$, where the observable is stable for $t_{\min } / a \geq 4$. Note that as we increase $t_{\min } / a$ or $\Delta \tau_{\min } / a$, fewer data are available to fit, and, consequently, the results become less precise. Thus, as in Ref. [20], we have demonstrated control over excited-state contamination when extracting matrix elements from staggered-baryon correlators.

TABLE II. Summary of the nominal fit range parameters. $t$ is the source-sink separation time, and $\tau$ is the current insertion time. $t_{\min }$ and $t_{\max }$ are the minimum and maximum source-sink separation included in the nominal fits; $\Delta \tau_{\min }$ is the minimum time after the current insertion time that we include in the threepoint fits.

\begin{tabular}{lcc}
\hline \hline Correlator & Fit parameter & Nominal value \\
\hline Two-point & $t_{\min } / a$ & 5 \\
& $t_{\max } / a$ & 13 \\
Three-point & $\Delta \tau_{\min } / a$ & 3 \\
& $t_{\max } / a$ & 13 \\
\hline \hline
\end{tabular}



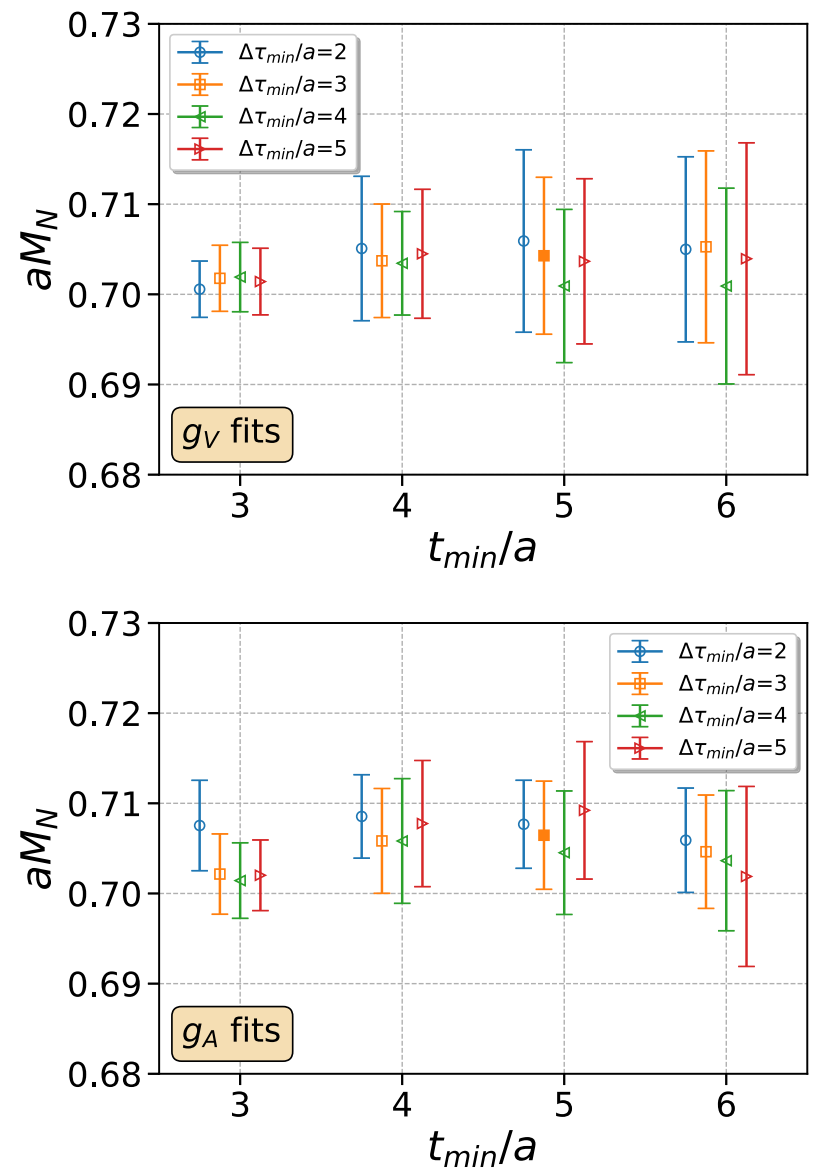

FIG. 4. The stability plot for the extracted nucleon mass, $a M_{N}$, as a function of $t_{\min }$ and $\Delta \tau_{\min }$, obtained from either the $g_{V}$ (top) or the $g_{A}$ (bottom) fits. The definitions of $t_{\min }$ and $\Delta \tau_{\min }$ are described in the text. The maximum source-sink separation time is fixed at $t_{\max }=13 a$ for all correlators. The solid squares are the nominal fit results, and all uncertainties are estimated with 1000 bootstrap samples.

\section{RESULTS}

In this section, we present our Bayesian fitting results and our final renormalized values for the nucleon charges $g_{V}$ and $g_{A}$. All fitting errors are estimated from 1000 bootstrap samples. We take correlations into account by using the same bootstrap samples for both $\tilde{g}_{V}$ and $\tilde{g}_{A}$.

\section{A. Nucleon mass}

In Fig. 6, we plot the extracted posterior fitted value for the nucleon mass from simultaneous fits of both smearings of the optimized two-point correlator and three-point correlator of a given current. We also plot the nucleonoptimized effective masses. These effective-mass data are identical to the $t_{0} / a=6$ data shown in Fig. 1. The greenshaded bands are the posterior estimates with the $g_{A}$ threepoint correlators, while the yellow-shaded bands are with the $g_{V}$ three-point correlators. We obtain $a M_{N}=0.707(6)$ from the $g_{A}$ fit and $a M_{N}=0.704(9)$ from the $g_{V}$ fit.
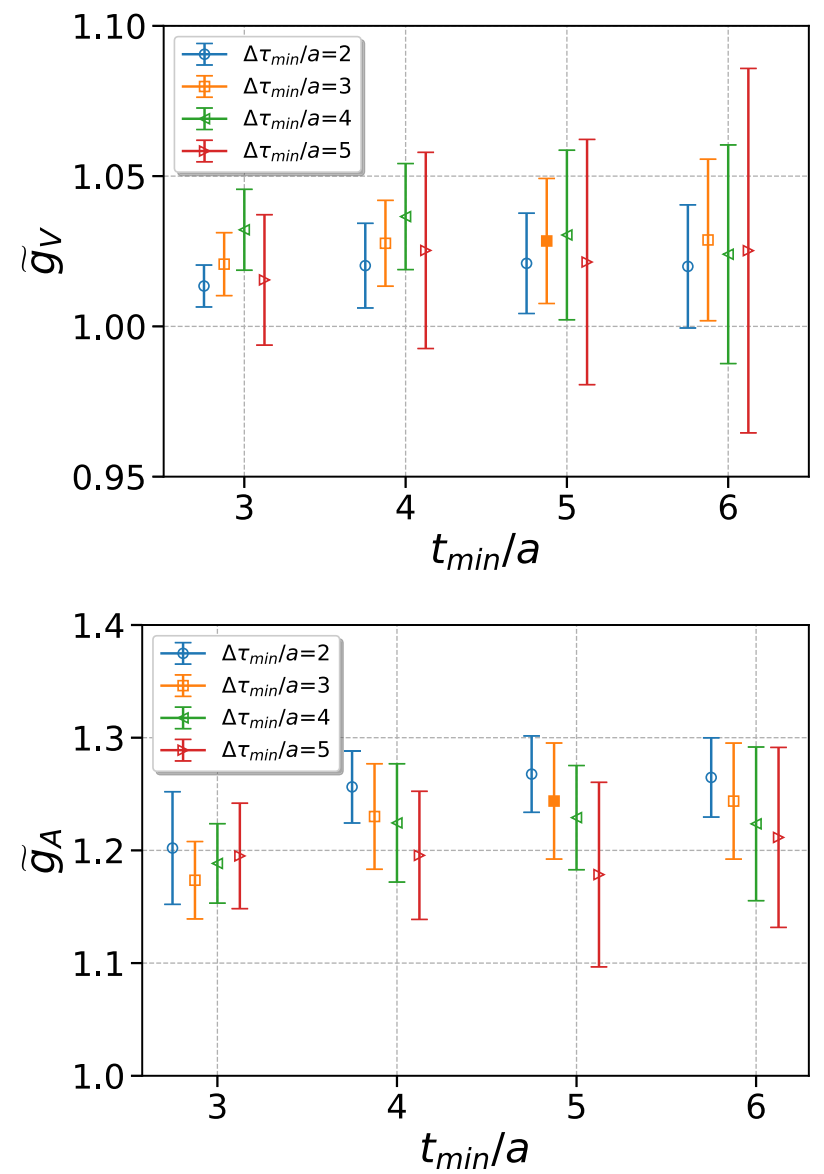

FIG. 5. The stability plot for the bare vector charge, $\tilde{g}_{V}$ (top), and bare axial charge, $\tilde{g}_{A}$ (bottom), as a function of $t_{\min }$ and $\Delta \tau_{\min }$. See the caption of Fig. 4 for further details.

There are some notable features in our fits. First, the $g_{V}$ fit has larger posterior uncertainties than the $g_{A}$ fit. Both fits include the same information from the two-point correlators, so the difference must arise from the three-point correlators. As one can see in Fig. 2, the $g_{V}$ three-point correlators are less sensitive to the Wuppertal smearing than the $g_{A}$ correlators. On the other hand, the $g_{V}$ three-to-two-point correlator ratios show remarkably little curvature, even at the early times. This behavior implies that the vector three-point correlators become quickly saturated by the ground state, and therefore provide limited additional information about the overlap factors and masses than what is contained in the two-point correlators. The $g_{A}$ data do not share these features, and thus contain additional information about the two-point posteriors. This explains why the $g_{V}$ fit has a less precise nucleon mass than the $g_{A}$ fit.

For these reasons, we quote the posterior nucleon mass from the $g_{A}$ fits as the nominal result, which has value

$$
a M_{N}=0.707(6), \quad M_{N}=1141(10) \mathrm{MeV},
$$

where the error shown is statistical only. It is crucial to bear in mind that this result is for a lattice spacing of 

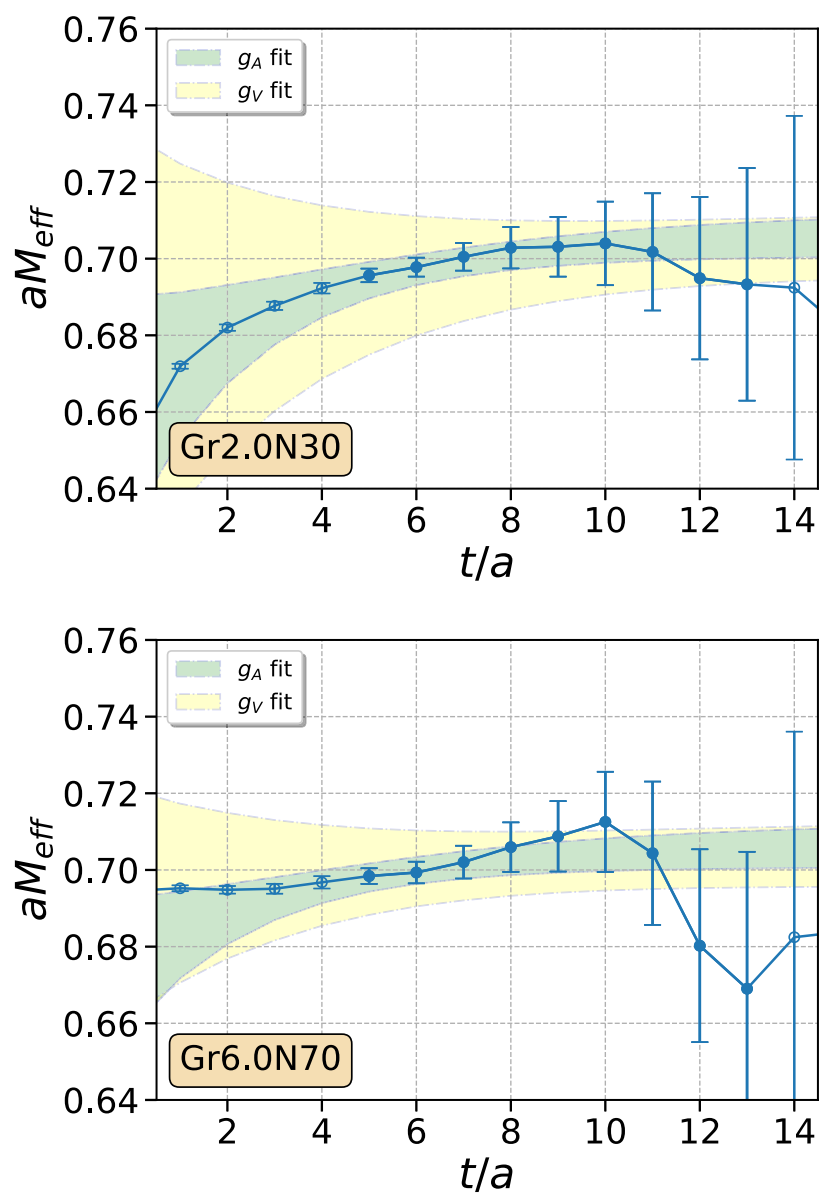

FIG. 6. Nominal fit results for the effective masses of the optimized correlators as a function of source-sink separation time $t$. The open circles are excluded from the fits. Correlators are labeled by their Wuppertal smearing parameters, with rms radii of $0.2 \mathrm{fm}$ (Gr2.0N30) or $0.6 \mathrm{fm}$ (Gr6.0N70). We perform simultaneous fits to two-point correlators with either the $g_{A}$ or the $g_{V}$ three-point correlators. Both sets of Wuppertal smeared correlators are included in each fit. The green and yellow shading shows the $1 \sigma$ bands from fits with either $g_{A}$ or $g_{V}$, respectively.

$a=0.1222(3) \mathrm{fm}$ and pion mass of $M_{\pi}=305 \mathrm{MeV}$ [29]. For comparison, a fit including only the two-point correlators yields $a M_{N}=0.704(9)$, which is identical to the posterior of the fit with $g_{V}$.

In Ref. [20], we computed the nucleon mass at the same lattice spacing but with a physical pion mass, obtaining $M_{N}=960(9) \mathrm{MeV}$. The difference between these two masses is $\Delta M_{N}=181(13) \mathrm{MeV}$, assuming uncorrelated statistical errors. Given that the pion mass difference between these two ensembles is about $170 \mathrm{MeV}, \Delta M_{N}$ agrees within $1 \sigma$ with the empirical observation that $M_{N}=$ $800 \mathrm{MeV}+M_{\pi}$ within a few percent [34].

\section{B. Nucleon $\boldsymbol{g}_{\boldsymbol{V}}$ and $\boldsymbol{g}_{\boldsymbol{A}}$ charges}

In Figs. 7 and 8, we plot the optimized $g_{V}$ and $g_{A}$ threeto-two-point correlator ratios as a function of source-sink
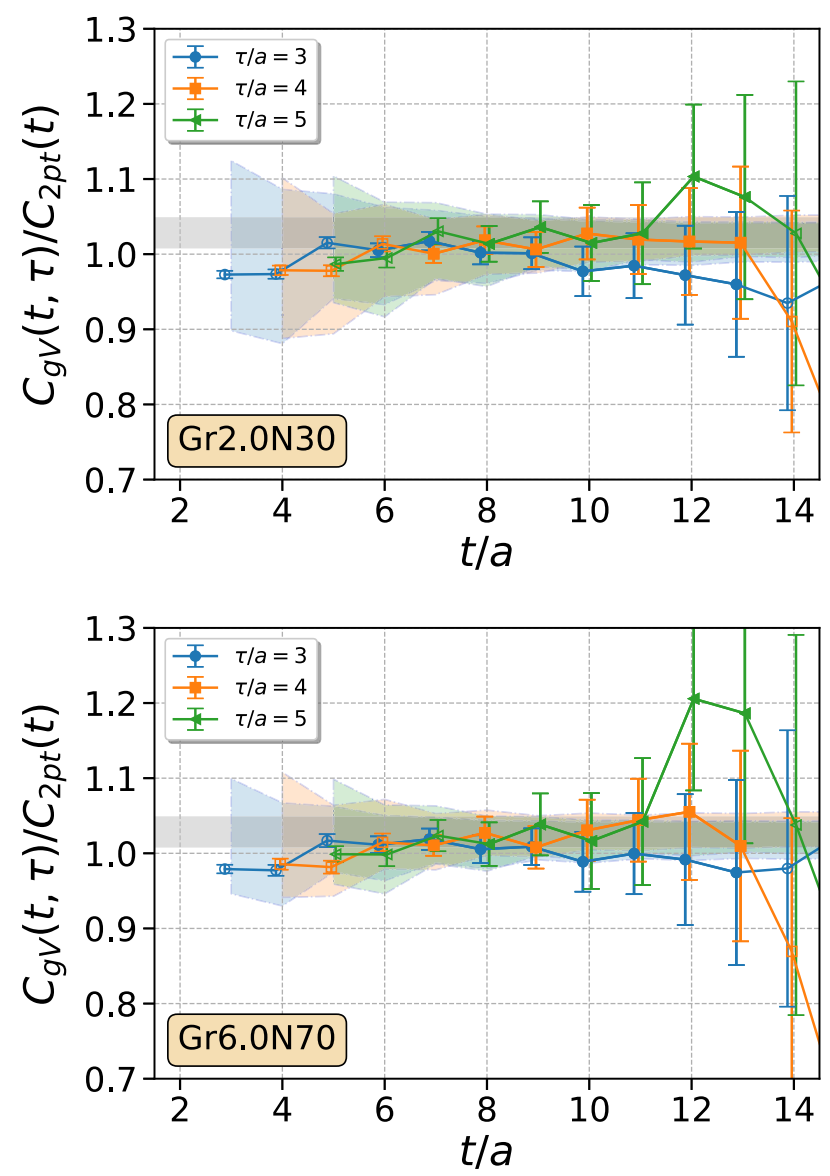

FIG. 7. Nominal fit results for the optimized three-to-two-point correlator ratio as a function of source-sink separation time $t$ and current insertion time $\tau$. In the limits $\tau, t-\tau \rightarrow \infty$, the optimized three-to-two-point correlator ratios converge to the bare axial charge $\tilde{g}_{V}$. Data points from different current insertion times, $\tau$, are slightly displaced for clarity. The filled data points are included in the nominal fit. Correlators are labeled by their Wuppertal smearing parameters with rms radii of $0.2 \mathrm{fm}$ (Gr2.0N30) or $0.6 \mathrm{fm}$ (Gr6.0N70). The $1 \sigma$ error bands for the different $\tau$ 's are shown in blue, orange, and green, and the $1 \sigma$ error band for the $\tilde{g}_{V}$ posterior is shown in gray.

separation $t$. The raw data are identical to the right-hand plots of Figs. 2 and 3. The posterior fit results are superimposed as gray bands. In the limits $\tau, t-\tau \rightarrow \infty$, the data points are seen to converge to these posteriors. It should be emphasized, however, that the ratio data points are shown only for illustration: we perform direct fits to the optimized correlators, as discussed in Sec. V, in order to obtain results, namely

$$
\begin{aligned}
& \tilde{g}_{V}=1.03(2), \\
& \tilde{g}_{A}=1.24(5) .
\end{aligned}
$$

It should be mentioned that the $g_{V}$ and $g_{A}$ fits have some different features. First, the residual oscillations from the 

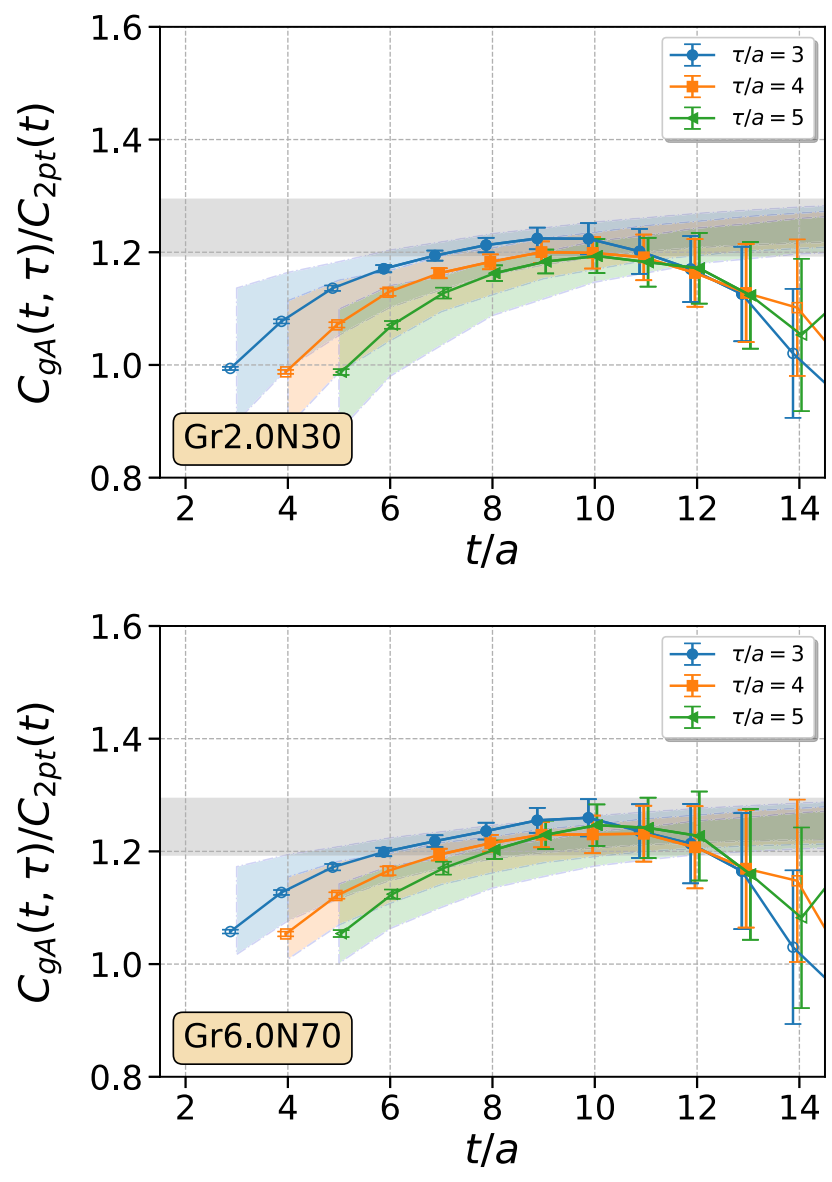

FIG. 8. Similar to Fig. 7, but for the axial-vector three-point correlators $\tilde{g}_{A}$.

parity partner matrix element are noticeable in the $g_{V}$ fit. Second, the $g_{V}$ data turn out to be relatively insensitive to the Wuppertal smearing radius. Both of these features can be observed in Fig. 7. This highlights that there are less uncorrelated data available with which to extract $g_{V}$ as compared to $g_{A}$. In contrast, we observe that the vector correlators in Fig. 7 contain less positive parity excitedstate contamination at early times than the axial-vector correlators in Fig. 8. As such, since the oscillations turn out to be easier to constrain and there is less contribution from the same parity excited states, we obtain a more precise estimate for $\tilde{g}_{V}$ than for $\tilde{g}_{A}$.

As discussed in Sec. II B, the remnant chiral symmetry enforces $Z_{A}=Z_{V}+\mathrm{O}\left(a m_{q}\right)^{2}$. Therefore, the ratio of bare charges is renormalized, and we obtain a value of

$$
\frac{g_{A}}{g_{V}}=\frac{\tilde{g}_{A}}{\tilde{g}_{V}}=1.21(5)
$$

where the correlation between $\tilde{g}_{A}$ and $\tilde{g}_{V}$ is taken into account via bootstrapping. We can also obtain $Z_{V}$ by imposing current conversation on a pseudoscalar meson vector-current matrix element [26]. Then the renormalized charges are

$$
\begin{aligned}
& g_{V}=Z_{V} \tilde{g}_{V}=1.02(2), \\
& g_{A}=Z_{A} \tilde{g}_{A}=1.23(5),
\end{aligned}
$$

based on $Z_{V}=Z_{A}=0.991(1)[26]$.

\section{DISCUSSION AND CONCLUSIONS}

We have presented two key results in this work. First, we have shown how to analytically relate the staggered nucleonlike matrix elements with nontrivial tastes to the physical nucleon matrix elements. This step is crucial for our ongoing program of extracting high-precision nucleon results from staggered fermions. The general procedure, which can be applied to any staggered baryon matrix element, is outlined in Appendixes A and B. Specifically, for the nucleon charges $g_{V}$ and $g_{A}$, we summarize our key results for the zero-momentum isovector (axial) vector three-point correlators in Eqs. (2.3) and (2.4). These equations explicitly show the nontrivial normalizations needed to relate the nucleonlike matrix elements to their physical counterparts. Our successful computation of $g_{V}$ and $g_{A}$ shows that continued use of the 16 irrep of the staggered symmetry group GTS is feasible, which is convenient because the 16 contains a single nucleonlike taste in the spectrum [20].

This finding is encouraging, because the additional complexity of staggered baryons, compared with staggered mesons is probably the reason staggered-baryon matrix elements have not been explored until now. There are as many meson tastes (16) as bosonic irreps of GTS. As such, each staggered meson interpolating operator excites only a single taste of meson. In contrast, there are $64=4^{3}$ different tastes of a staggered baryon, yet only three unique irreps of GTS, denoted 8, 8', and 16 after their dimensions. Consequently, there are not enough unique components of these irreps to accommodate all 64 tastes of baryons, and more than one taste of the same baryon can appear in each irrep's tower of states. Choosing an irrep with only one nucleon taste simplifies the correlator analysis and, as we have shown in this paper, allows for accurate and precise results for nucleon matrix elements.

The second key result of this work is demonstrating the practicality of staggered baryons by computing the isovector nucleon vector and axial-vector charges. For this purpose, we choose a single ensemble with $a \approx 0.12 \mathrm{fm}$, $2+1+1$ flavors in the sea, and, when using identical sea and valence HISQ quarks, $M_{\pi}=305 \mathrm{MeV}$. With approximately 7000 measurements and techniques designed to handle staggered correlators, we find few-percent statistical uncertainty. Our final values for $g_{V}, g_{A}$, and $g_{A} / g_{V}$ on this ensemble are 


$$
\begin{aligned}
& g_{V}=1.02(2), \\
& g_{A}=1.23(5), \\
& \frac{g_{A}}{g_{V}}=1.21(5) .
\end{aligned}
$$

The conservation of the vector charge, $g_{V}=1$, is a nontrivial verification of our methodology.

As discussed in Sec. V, we include two positive-parity states and one negative-parity state in our fit function. The number of matrix elements included in the fit grows quadratically as a function of the states included. With more precise data, we could constrain more matrix elements. Alternatively, we could also impose tighter priors on the transition matrix elements and overlap factors, for example, with the empirical Bayes method [24]. This proof-of-concept study does not attempt a full calculation with all errors included, so we leave exploration of those options for future work.

The same ensemble has been used by both the CalLat [23] and PNDME [24] Collaborations in their calculations of $g_{V}$ and $g_{A}$. CalLat uses Möbius domain-wall fermions for the valence quarks, while PNDME uses Wilson fermions with the clover action. CalLat defines $Z_{V}$ by demanding $g_{V}=Z_{V} \tilde{g}_{V}=1$ and uses the remnant chiral symmetry to set $Z_{A}=Z_{V}$. They then quote $\tilde{g}_{V}=1.021(2)$ and $g_{A}=1.21(1)$. PNDME determines $Z_{V}$ and $Z_{A}$ independently via the regularization-independent symmetric momentum-subtraction scheme, commonly known as RI-sMOM, and quote $g_{V}=0.97(2), g_{A}=1.21(4)$, and $g_{A} / g_{V}=1.25(2)$. Our result is consistent with both, despite the different choices of valence-quark formulation.

Several further lattice-QCD calculations of $g_{A}$ exist in the literature [35]. Calculations performed on $2+1+1$ ensembles include those from CalLat [23] and PNDME [24], discussed above, and one with improved Wilson sea and valence quarks [36]. Additional determinations of $g_{A}$ on $2+1$ ensembles have been carried out [37-46], as well as others without the strange sea [36,47-54]. It would be nice to make a detailed comparison of the computational costs of all these calculations, but not all relevant information has been published. Thus, the calculations performed on the same MILC ensemble provide the most apt comparison.

With an eye toward subpercent determinations of the axial charge, it is instructive to compare how the precision on $g_{A}$ is influenced by each collaboration's data and methodology, limiting our considerations to the three calculations on the same HISQ ensemble. The three analyses share a few common aspects. First is the use of eight sources (with high-precision solutions of the Dirac equation) per gauge-field configuration, so the raw statistics are about the same. Second, the time range of the central fits for the two-point correlators turns out to be the same: $t_{\max }+1-t_{\min }=8$. Third, all three collaborations simultaneously fit a correlator containing the matrix element with the two-point correlators. Last, PNDME and we use time ranges in the central fits of the threepoint correlators, such that there are 21 data points in the fit.

In addition, each collaboration employs techniques to improve the signal. We have two smeared sinks and start with $4 \times 4$ matrix correlators, following from the four operator classes for our choice of staggered irrep. We apply the GEVP to the $4 \times 4$ matrix for each smearing radius to find the optimal source and sink operators for the nucleon. PNDME increases statistics via the truncatedsolver method with bias correction $[55,56]$. CalLat reduces noise with an $a$-independent number of steps of a gradient flow [57]. In the future, we could easily take advantage of the truncated-solver method, while the gradient flow would prevent us from using numerous technical results from the Fermilab Lattice and MILC Collaborations, such as latticespacing and renormalization-factor determinations.

A more striking difference is CalLat's introduction of the currents into a propagator in a way inspired by the Feynman-Hellmann theorem [58]. A key feature of the technique is that instead of a three-point function, the matrix element lies within another two-point function. Thus, the CalLat method requires a fit to a single time variable instead of two; indeed the matrix element pops out of a fit to the ratio of the two two-point correlators.

In the end, the relative precision on $g_{A}$ is quoted as $1 \%$, $3 \%$, and $4 \%$ for CalLat [23], PNDME [24], and this work, respectively. One should bear in mind, however, the effective number of components per site, which are four for Wilson fermions, eight for staggered fermions (corresponding to the corners of the unit cube), and $4 L_{5}$ for domain-wall fermions (where $L_{5}$ is the extent of the fifth dimension; $L_{5}=8$ in Ref. [23]). Taking the number of components into account but ignoring algorithmic speedups from the code or specific features of each action, the cost for a given precision is roughly the same. It would, therefore, be interesting to explore the truncated-solver and Feynman-Hellmann-inspired methods with staggered fermions.

For future reference, we estimate the total cost of this calculation to be 20,000 node hours of the LQ1 cluster at Fermilab, of which about $90 \%$ is used for propagator solves, $5 \%$ for Wuppertal smearing, and 5\% for correlator tie-ups. Each node on LQ1 consists of two twenty-core Intel Xeon Gold 6248 Processors, and the nodes are interconnected via an Intel EDR Omni-Path network. In this work, however, all calculations ran on a single node.

This work sets the foundation needed to continue a program of precise nucleon form-factor calculations. Calculations of the vector and axial-vector form factors at nonzero momentum transfer are indeed underway on the same ensemble as used here. Further, we have started computing $g_{V}$ and $g_{A}$ on the same ensembles used in 
Ref. [20]. These ensembles have physical pion masses and a range of lattice spacings to enable a continuum extrapolation.

\section{ACKNOWLEDGMENTS}

We are grateful to the MILC Collaboration for the use of the source code adapted to generate the correlators in this study and for permission to use their $2+1+1$-flavor gauge-field ensemble. Computations for this work were carried out on facilities of the USQCD Collaboration, which are funded by the Office of Science of the U.S. Department of Energy. This manuscript has been authored by Fermi Research Alliance, LLC, under Contract No. DEAC02-07CH11359 with the U.S. Department of Energy, Office of Science, Office of High Energy Physics. Brookhaven National Laboratory is supported by the U.S. Department of Energy under Contract No. DESC0012704. Additional support was provided under U.S. DOE Contract No. DE-SC00190193.

\section{APPENDIX A: RELATING STAGGERED-QCD MATRIX ELEMENTS TO QCD MATRIX ELEMENTS}

Lattice gauge theory with staggered fermions can be thought of as an extension of QCD with four degenerate flavors, called tastes, for each quark. The associated taste symmetry allows for many more composite states which can have nontrivial taste structures. We call states that have nontrivial taste "baryonlike" states, to distinguish from the physical single-taste baryons. In this work, we focus on the nucleon and restrict ourselves to that case going forward. The nucleonlike states can be mapped onto the physical nucleon states through appropriate flavor-taste symmetry transformations. This allows the freedom to choose which nucleonlike state to study in order to extract observables. As highlighted in Ref. [20], the two-point correlator data constructed from nucleonlike states are easier to analyze than their physical counterparts due to the smaller multiplicity of tastes in the spectrum. However, one needs the mapping from the specific nucleonlike state to the physical state.

We use isospin- $\frac{3}{2}$, GTS-16 nucleonlike interpolating operators to extract nucleon observables, since the spectrum contains only a single nucleonlike state. The relationship between the nucleonlike matrix elements and the physical nucleon matrix elements is, unfortunately, not at all transparent. In this and the following Appendixes, we will establish the relationship between the 16-irrep nucleonlike matrix elements and the single-taste physical nucleon matrix elements.

Bailey [19] inferred the spectrum of staggered baryons by subducing nucleonlike representations of the full $\mathrm{SU}(8)_{\mathrm{FT}}$ flavor-taste symmetry of the continuum limit into GTS. We expand that work to matrix elements. Specifically, we will demonstrate how one can apply the generalized WignerEckart theorem to SU(4) and relate the lattice nucleonlike matrix elements to the physical tasteless nucleon matrix elements through appropriate normalization factors, which are generalized Clebsch-Gordan coefficients. The procedure outlined here can be applied to any staggered baryon matrix elements in any $\mathrm{SU}\left(n_{f}\right) \times$ GTS flavor-taste irrep.

Following the notation from Ref. [20], we first determine the continuum quantum numbers of the nucleonlike states that subduce into the 16 irrep of GTS. This step is needed for the generalized Wigner-Eckart theorem. We focus on the continuum symmetry group $\mathrm{SU}(2)_{S} \times \mathrm{SU}(8)_{\mathrm{FT}}$, where $\mathrm{SU}(2)_{S}$ is the spin symmetry and $\mathrm{SU}(8)_{\mathrm{FT}}$ is the flavor $(F)$ and taste $(T)$ symmetry for two equal-mass flavors. This group breaks on a discrete lattice to the unbroken flavor symmetry subgroup $\mathrm{SU}(2)_{F}$ and the GTS $[18,19]$. The GTS can be decomposed into [20,59]

$$
\mathrm{GTS}=\left(\left(\mathbb{Q}_{8} \rtimes \mathrm{SW}_{3}\right) \times \mathrm{D}_{4}\right) / \mathbb{Z}_{2},
$$

where $\mathbb{Q}_{8}$ is generated by the discrete taste transformations $\left\{\Xi_{12}, \Xi_{23}\right\}, \mathrm{SW}_{3}$ by the cubic rotations $\left\{R_{12}, R_{23}\right\}$, and $\mathrm{D}_{4}$ by the discrete taste and spatial inversion transformations $\left\{\Xi_{123}, I_{S}\right\}$. (These symbols are all defined in the Appendix of Ref. [20].)

The subgroup chain we work with is ${ }^{1}$

$$
\begin{aligned}
& \mathrm{SU}(2)_{S} \times \mathrm{SU}(8)_{\mathrm{FT}} \times P \supset \mathrm{SU}(2)_{S} \times \mathrm{SU}(2)_{F} \times \mathrm{SU}(4)_{T} \times P \\
& \supset \mathrm{SU}(2)_{S} \times \mathrm{SU}(2)_{F} \times \mathrm{SU}(2)_{\mathbb{Q}_{8}} \times \mathrm{SU}(2)_{\mathrm{D}_{4}} \times \mathrm{U}(1)_{\mathrm{D}_{4}} \times P \\
& \supset \mathrm{SU}(2)_{F} \times \mathrm{GTS} \times P,
\end{aligned}
$$

where $P=I_{S} \Xi_{4}$ becomes the usual parity operation in the continuum limit [18]. The factor $\mathrm{SU}(2)_{\mathrm{D}_{4}}$ on the second line arises from decomposing the $\mathrm{SU}(4)_{T}$ taste symmetry onto a discrete lattice, which leads to the factor $D_{4}$ in Eq. (A1), combined with the $\mathrm{U}(1)_{\mathrm{D}_{4}}$ phase factor. Note that in Ref. [20] we omitted the $\mathrm{U}(1)_{\mathrm{D}_{4}}$ factor, but here we make it explicit. The other groups are defined and explained in Ref. [20].

\section{Using shift symmetries to relate staggered correlators}

The goal is to assign continuum quantum numbers of $\mathrm{SU}(2)_{S} \times \mathrm{SU}(2)_{F} \times \mathrm{SU}(4)_{T}$ to each nucleonlike state created by every component of the 16 irrep. We begin by investigating the continuum quantum numbers of the simplest nucleonlike states created by the 16 irrep. Afterwards, we can use the lattice symmetry transformations to obtain the remaining components.

\footnotetext{
${ }^{1}$ Various $\mathbb{Z}_{N}$ quotient factors are often omitted for clarity. They are only necessary to avoid overcounting group elements [for example, $\left.\mathrm{SU}(4)_{T} \supset\left(\mathrm{SU}(2)_{\mathbb{Q}_{8}} \times \mathrm{SU}(2)_{\mathrm{D}_{4}}\right) / \mathbb{Z}_{2}\right]$.
} 
We can form nonvanishing two-point correlation functions by contracting any one of the 16 irrep components with the same component on a later time slice. One can then apply lattice rotations and shifts ${ }^{2}$ to show that these 16 twopoint correlators are identical in the ensemble average.

The 16 irrep components split into two sets of eight different components that reside on the eight corners of a cube (see the appendix of Ref. [20] for explicit constructions). The construction of nonvanishing three-point correlator data also depends on the current insertion. For the local vector and axial-vector currents we use in this work, the zero-momentum three-point correlators do not vanish if and only if the source and sink interpolators are identical. Correlators constructed from the same set of eight components can be related to each other with the lattice shift symmetries.

To summarize, this means that the nonvanishing twopoint correlators satisfy

$\sum_{\vec{x}}\left\langle B_{+\vec{M}}^{16}(\vec{x}, t) \bar{B}_{+\vec{M}}^{16}(0)\right\rangle=\sum_{\vec{x}}\left\langle B_{s \vec{N}}^{16}(\vec{x}, t) \bar{B}_{s \vec{N}}^{16}(0)\right\rangle$,

where the superscript denotes the 16 irrep operators, $\vec{M}$ and $\vec{N}$ are equal to any one of the eight corners of the cube, and $s= \pm 1$ are the eigenvalues of the lattice rotation $R_{12}$ for $\vec{M}=\vec{N}=\overrightarrow{0}$. The notation is defined in detail in Ref. [20].

We are using local currents $J=V, A$ in this work, so the nonvanishing three-point correlators satisfy

$$
\begin{aligned}
\sum_{\vec{x}, \vec{y}} & \left\langle B_{s \vec{M}}^{16}(\vec{x}, t) J(\vec{y}, \tau) \bar{B}_{s \vec{M}}^{16}(0)\right\rangle \\
& =\mathcal{S}_{J}(\vec{N}-\vec{M}) \sum_{\vec{x}, \vec{y}}\left\langle B_{s \vec{N}}^{16}(\vec{x}, t) J(\vec{y}, \tau) \bar{B}_{s \vec{N}}^{16}(0)\right\rangle
\end{aligned}
$$

where $\mathcal{S}_{J}(\vec{A})= \pm 1$ is a sign factor that depends on both $J$ and $\vec{A}$. Its specific value can be determined by applying a lattice shift symmetry transformation between $\vec{M}$ and $\vec{N}$. For the currents used in this work, it is identical to the sign factor appearing in the construction of the staggered current $J$. For example, $\mathcal{S}_{V}(\vec{A})=(-1)^{\left(A_{x}+A_{y}+A_{z}\right) / a}$ for the $\gamma_{4} \otimes \xi_{4}$ vector current and $\mathcal{S}_{A}(\vec{A})=(-1)^{A_{z} / a}$ for the $\gamma_{z} \gamma_{5} \otimes \xi_{z} \xi_{5}$ axial current. The currents and phase factors are also defined in Eqs. (2.5) and (2.6). For a general current (other than the local currents used here), however, it might be necessary to have different interpolating operators at the source and sink. In that case, the phase factors in the general version of Eq. (A4) would still be obtained from the lattice shift symmetries.

\footnotetext{
${ }^{2}$ We use the convention of staggered phases, $\eta_{1}(x)=(-1)^{x_{4}}$, $\eta_{2}(x)=(-1)^{x_{4}+x_{1}}, \eta_{3}(x)=(-1)^{x_{4}+x_{1}+x_{2}}$, and $\eta_{4}(x)=1 \quad[60]$, which affects the phases appearing in the lattice rotations and shifts.
}

Going forward, it is sufficient to study the correlator with component $\vec{N}=0$ located at the origin of the staggered unit cube, $\sum_{\vec{x}} B_{+\overrightarrow{0}}^{16}(\vec{x}, t)$. Then, owing to Eq. (A4), the other seven components follow immediately.

The quantum numbers of the nucleonlike states created by $\sum_{\vec{x}} B_{ \pm \overrightarrow{0}}^{16}(\vec{x}, t)$ will be denoted as $\left|\left[\frac{3}{2}, \frac{3}{2}\right]_{F}[16, \pm \overrightarrow{0}]_{\mathrm{GTS}}\right\rangle$. The first bracket gives the unbroken $\mathrm{SU}(2)_{F}$ flavor quantum numbers, which here has total and $z$-component isospins $\frac{3}{2}$, and the second bracket denotes the 16 irrep with the eigenvalues of $R_{12}$.

\section{Quantum numbers of nucleonlike states}

Next, we must find a convenient basis for the continuum nucleonlike states and then subduce them down to the $\left|\left[\frac{3}{2}, \frac{3}{2}\right]_{F}[16, \pm \overrightarrow{0}]_{\mathrm{GTS}}\right\rangle$ lattice states. From Eq. (A2), we want to track the quantum numbers of $\mathrm{SU}(2)_{S} \times \mathrm{SU}(2)_{F} \times$ $\mathrm{SU}(4)_{T}$ and may ignore the passive phase $\mathrm{U}(1)_{\mathrm{D}_{4}}$ and parity $P=+1$ factors. From the group subduction presented in Refs. [19,20], the 16 irrep is subduced from the continuum spin-flavor-taste irrep via

$$
\begin{aligned}
& \mathrm{SU}(2)_{S} \times \mathrm{SU}(2)_{F} \times \mathrm{SU}(4)_{T} \\
& \quad \supset \mathrm{SU}(2)_{S} \times \mathrm{SU}(2)_{F} \times \mathrm{SU}(2)_{\mathbb{Q}_{8}} \times \mathrm{SU}(2)_{\mathrm{D}_{4}},
\end{aligned}
$$

$$
\begin{aligned}
& \left(\frac{1}{2}, \frac{3}{2}, 20_{M}\right) \\
& \quad \rightarrow\left(\frac{1}{2}, \frac{3}{2}, \frac{1}{2}, \frac{3}{2}\right) \oplus\left(\frac{1}{2}, \frac{3}{2}, \frac{3}{2}, \frac{1}{2}\right) \oplus\left(\frac{1}{2}, \frac{3}{2}, \frac{1}{2}, \frac{1}{2}\right) .
\end{aligned}
$$

Here we have adopted a convention that labels non-SU(2) group irreps by their dimensions and subscript $M$ (mixed), $S$ (symmetric), or $A$ (antisymmetric). The irreps of $\mathrm{SU}(2)$ are denoted with standard spin notation.

The task of classifying a general irrep of SU(4) amounts to finding the maximal set of commuting operators and uniquely labeling the states by their eigenvalues; for a general SU(4) irrep, there are six eigenvalues to classify [61]. Because there are no degenerate irreps when decomposing any of the irreps in this work from $\mathrm{SU}(4)$ into $\mathrm{SU}(2) \times \mathrm{SU}(2)$, we can use the eigenvalues of the pair of $\mathrm{SU}(2)$ factors to identify SU(4) states. Therefore, only four of those six eigenvalues are necessary to completely characterize the states. As such, the four eigenvalues of each state can be uniquely identified with two pairs of the $\left|L^{2}, L_{z}\right\rangle$ quantum numbers.

Given Eq. (A6), we notice that $20_{M} \rightarrow\left(\frac{1}{2}, \frac{1}{2}\right) \oplus\left(\frac{3}{2}, \frac{1}{2}\right) \oplus$ $\left(\frac{1}{2}, \frac{3}{2}\right)$. We seek to find the four quantum numbers for the states after decomposition of the $20_{M}$ irrep of $\mathrm{SU}(4)_{T}$ into the subgroup $\mathrm{SU}(2)_{\mathbb{Q}_{8}} \times \mathrm{SU}(2)_{\mathrm{D}_{4}}$. We write the continuum nucleonlike states kets 


$$
\left|\left[\frac{1}{2}, m^{S}\right]_{S}\left[\frac{3}{2}, \frac{3}{2}\right]_{F}\left[j^{\mathbb{Q}_{8}}, m^{\mathbb{Q}_{8}}\right]_{\mathbb{Q}_{8}}\left[j^{\mathrm{D}_{4}}, m^{\mathrm{D}_{4}}\right]_{\mathrm{D}_{4}}\right\rangle .
$$

Each bracket represents the standard spin quantum numbers of one of the $\mathrm{SU}(2)$ group factors, distinguished by the superscripts and subscripts: $S$ (spin), $F$ (flavor), $\mathbb{Q}_{8}\left[\mathrm{SU}(2)_{\mathbb{Q}_{8}}\right]$, and $\mathrm{D}_{4}\left[\mathrm{SU}(2)_{\mathrm{D}_{4}}\right]$. This ket serves as the irrep basis for both $\mathrm{SU}(2)_{S} \times \mathrm{SU}(2)_{F} \times \mathrm{SU}(4)_{T}$ and $\mathrm{SU}(2)_{S} \times \mathrm{SU}(2)_{F} \times \mathrm{SU}(2)_{\mathbb{Q}_{8}} \times \mathrm{SU}(2)_{\mathrm{D}_{4}}$.

\section{Matching the continuum and lattice nucleonlike states}

Now that we have established an appropriate basis for the nucleonlike states, both on the lattice and in the continuum, we are ready to match the two sets. In particular, we are interested in which linear combination of states from Eq. (A7) combine to subduce into the lattice states $\left|\left[\frac{3}{2}, \frac{3}{2}\right]_{F}[16, \pm \overrightarrow{0}]_{\text {GTS }}\right\rangle$ of interest. For the 16 irrep nucleonlike states, we have shown in Ref. [20] that $j^{\mathbb{Q}_{8}}=\frac{3}{2}$ and $j^{\mathrm{D}_{4}}=\frac{1}{2}$. Consequently, we only need to determine $m^{S}, m^{\mathbb{Q}_{8}}$, and $m^{\mathrm{D}_{4}}$.

We start with determining $m^{\mathrm{D}_{4}}$ of $\mathrm{SU}(2)_{\mathrm{D}_{4}}$ from Eq. (A7). To do so, it is illuminating to study the decomposition

$$
\mathrm{SU}(2)_{\mathrm{D}_{4}} \times \mathrm{U}(1)_{\mathrm{D}_{4}} \times P \rightarrow\left\{I_{S}\right\},
$$

where $\left\{I_{S}\right\}$ is the group generated by the lattice spatial inversion. As Eq. (A8) shows, $I_{S}$ receives contributions from three different factors: the taste factor $\mathrm{SU}(2)_{\mathrm{D}_{4}}$, a phase factor $e^{-i \pi / 2}=-i$ from $\mathrm{U}(1)_{\mathrm{D}_{4}}$ to match the eigenvalues of $I_{S}$, and the continuum-limit parity $P=I_{S} \Xi_{4}$. For the spin- $\frac{1}{2}$ irreps of $\mathrm{SU}(2)_{\mathrm{D}_{4}}$, which include the 16 irrep nucleons [20], the matrix representation of $I_{S}$ is the tensor product of those three factors

$$
e^{i \sigma_{3} \pi / 2} \otimes e^{-i \pi / 2} \otimes+1=\left[\begin{array}{cc}
1 & 0 \\
0 & -1
\end{array}\right]=\sigma_{3}=I_{S},
$$

where $\sigma_{3}$ is the third Pauli matrix. The representation in Eq. (A9) can be mapped onto the groups in Eq. (A8). The first factor arises from the 180 degrees rotation in the " $x-y$ plane" of the spin- $\frac{1}{2}$ representation of $\mathrm{SU}(2)_{\mathrm{D}_{4}}$, the second $e^{-i \pi / 2}$ phase is from $\mathrm{U}(1)_{\mathrm{D}_{4}}$, and the +1 is from parity. As can be seen from Eq. (A9), for the spin- $\frac{1}{2}$ irrep of $\mathrm{SU}(2)_{\mathrm{D}_{4}}$, the $I_{S}$ matrix admits \pm 1 eigenvalues which arise from the $m^{\mathrm{D}_{4}}= \pm \frac{1}{2}$ components of $\sigma_{3}$. Since the nucleon is a positive-parity state with $I_{S}=1$, we assign $m^{\mathrm{D}_{4}}=\frac{1}{2}$ to the $\left|\left[\frac{3}{2}, \frac{3}{2}\right]_{F}[16, \pm \overrightarrow{0}]_{\text {GTS }}\right\rangle$ lattice states.

We now consider the quantum numbers of $m^{S}$ and $m^{\mathbb{Q}_{8}}$. The 16 irrep components can be labeled by the irreps of $\mathrm{W}_{3}=\mathrm{SW}_{3} \times\left\{1, I_{S}\right\}$, where $\mathrm{SW}_{3}$ is the cubic rotation group, as [18]

$$
16 \rightarrow E^{+} \oplus E^{-} \oplus T_{1}^{+} \oplus T_{1}^{-} \oplus T_{2}^{+} \oplus T_{2}^{-},
$$

where $E$ is the two-dimensional irrep of $\mathrm{SW}_{3}, T_{1}$ and $T_{2}$ are the different three-dimensional irreps of $\mathrm{SW}_{3}$, and the superscripts show the eigenvalues of $I_{S}$. By applying lattice rotations to $\left|\left[\frac{3}{2}, \frac{3}{2}\right]_{F}[16, \pm \overrightarrow{0}]_{\mathrm{GTS}}\right\rangle$, we can show they belong to the two-dimensional $E^{+}$irrep of $\mathrm{W}_{3}$.

Subducing $\mathrm{SU}(2)_{\mathrm{SW}_{3}} \subset \mathrm{SU}(2)_{\mathbb{Q}_{8}} \times \mathrm{SU}(2)_{S}$ to the lattice angular momentum of $\mathrm{SW}_{3}$ is a problem common to all fermion formulations [62]. We can write the irrep components of $\mathrm{SU}(2)_{\mathrm{SW}_{3}}$ that subduce into $E$ as [62]

$$
\begin{aligned}
&\left|[2,0]_{\mathrm{SW}_{3}}\left[\frac{1}{2}, \frac{1}{2}\right]_{\mathrm{D}_{4}}\right\rangle \rightarrow\left|[16,+\overrightarrow{0}]_{\mathrm{GTS}}\right\rangle \\
& \rightarrow\left|\left[E^{+},+\right]_{\mathrm{W}_{3}}\right\rangle, \\
& \frac{1}{\sqrt{2}}\left(\left|[2,2]_{\mathrm{SW}_{3}}\left[\frac{1}{2}, \frac{1}{2}\right]_{\mathrm{D}_{4}}\right\rangle+\left|[2,-2]_{\mathrm{SW}_{3}}\left[\frac{1}{2}, \frac{1}{2}\right]_{\mathrm{D}_{4}}\right\rangle\right) \\
& \rightarrow\left|[16,-\overrightarrow{0}]_{\mathrm{GTS}}\right\rangle \rightarrow\left|\left[E^{+},-\right]_{\mathrm{W}_{3}}\right\rangle,
\end{aligned}
$$

where the irreps of $\mathrm{SU}(2)_{\mathrm{SW}_{3}}$ are again labeled by the total and $z$ component of angular momentum, and the arrows indicate the subduction from continuum to lattice states. $\left|\left[E^{+}, \pm\right]_{\mathrm{W}_{3}}\right\rangle$ is a state that transforms in the $E^{+}$irrep of $\mathrm{W}_{3}$ with $\mathrm{a}+1$ eigenvalue under spatial inversion and \pm 1 eigenvalue under rotation $R_{12}$. We identify $\mathrm{SU}(2)_{\mathrm{SW}_{3}}$ as the diagonal subgroup of $\mathrm{SU}(2)_{S} \times \mathrm{SU}(2)_{\mathbb{Q}_{8}}$ [18]. Then, by using the Clebsch-Gordan coefficients, the components are related as

$$
\begin{aligned}
& \left|[2,0]_{\mathrm{SW}_{3}}\right\rangle \\
& =\frac{1}{\sqrt{2}}\left(\left|\left[\frac{1}{2}, \frac{1}{2}\right]_{S}\left[\frac{3}{2},-\frac{1}{2}\right]_{\mathbb{Q}_{8}}\right\rangle+\left|\left[\frac{1}{2},-\frac{1}{2}\right]_{S}\left[\frac{3}{2}, \frac{1}{2}\right]_{\mathbb{Q}_{8}}\right\rangle\right)
\end{aligned}
$$

and

$$
\begin{aligned}
& \frac{1}{\sqrt{2}}\left(\left|[2,2]_{\mathrm{SW}_{3}}\right\rangle+\left|[2,-2]_{\mathrm{SW}_{3}}\right\rangle\right) \\
& \quad=\frac{1}{\sqrt{2}}\left(\left|\left[\frac{1}{2}, \frac{1}{2}\right]_{S}\left[\frac{3}{2}, \frac{3}{2}\right]_{\mathbb{Q}_{8}}\right\rangle+\left|\left[\frac{1}{2},-\frac{1}{2}\right]_{S}\left[\frac{3}{2},-\frac{3}{2}\right]_{\mathbb{Q}_{8}}\right\rangle\right) .
\end{aligned}
$$

Taking all the results of this Appendix together, we have

$$
\begin{aligned}
|16,+\overrightarrow{0}\rangle & \equiv \frac{1}{\sqrt{2}}\left(\left|\left[\frac{1}{2}, \frac{1}{2}\right]_{S}\left[\frac{3}{2}, \frac{3}{2}\right]_{F}\left[\frac{3}{2},-\frac{1}{2}\right]_{\mathbb{Q}_{8}}\left[\frac{1}{2}, \frac{1}{2}\right]_{\mathrm{D}_{4}}\right\rangle\right. \\
& \left.+\left|\left[\frac{1}{2},-\frac{1}{2}\right]_{S}\left[\frac{3}{2}, \frac{3}{2}\right]_{F}\left[\frac{3}{2}, \frac{1}{2}\right]_{\mathbb{Q}_{8}}\left[\frac{1}{2}, \frac{1}{2}\right]_{\mathrm{D}_{4}}\right\rangle\right) \\
& \rightarrow\left|\left[\frac{3}{2}, \frac{3}{2}\right]_{F}[16,+\overrightarrow{0}]_{\mathrm{GTS}}\right\rangle,
\end{aligned}
$$




$$
\begin{aligned}
|16,-\overrightarrow{0}\rangle & \equiv \frac{1}{\sqrt{2}}\left(\left|\left[\frac{1}{2}, \frac{1}{2}\right]_{S}\left[\frac{3}{2}, \frac{3}{2}\right]_{F}\left[\frac{3}{2}, \frac{3}{2}\right]_{\mathbb{Q}_{8}}\left[\frac{1}{2}, \frac{1}{2}\right]_{\mathrm{D}_{4}}\right\rangle\right. \\
& \left.+\left|\left[\frac{1}{2},-\frac{1}{2}\right]_{S}\left[\frac{3}{2}, \frac{3}{2}\right]_{F}\left[\frac{3}{2},-\frac{3}{2}\right]_{\mathbb{Q}_{8}}\left[\frac{1}{2}, \frac{1}{2}\right]_{\mathrm{D}_{4}}\right\rangle\right) \\
& \rightarrow\left|\left[\frac{3}{2}, \frac{3}{2}\right]_{F}[16,-\overrightarrow{0}]_{\mathrm{GTS}}\right\rangle .
\end{aligned}
$$

Here, $|16, \pm \overrightarrow{0}\rangle$ have been introduced as shorthand notation for the continuum states for future reference.

\section{Quantum numbers of the current operators}

The last ingredient needed for the Wigner-Eckart theorem is the irreducible tensor current operator. In this work, we use the local isovector axial current, $A$, and local isovector vector current, $V$, which have spin tastes $\gamma_{z} \gamma_{5} \otimes \xi_{z} \xi_{5}$ and $\gamma_{4} \otimes \xi_{4}$, respectively. We will need their $\mathrm{SU}(2)_{S} \times \mathrm{SU}(2)_{F} \times \mathrm{SU}(4)_{T}$ quantum numbers, just as in the above sections.

The spin and flavor quantum numbers of the currents are straightforward. By construction, both currents have a total isospin equal to one, with $I_{z}=0$ components. $A$ is a spin-1 current with $S_{z}=0$, and $V$ is a spin scalar. The nontrivial part of the identification comes from mapping the quantum numbers of $\mathrm{SU}(2)_{\mathbb{Q}_{8}} \times \mathrm{SU}(2)_{\mathrm{D}_{4}}$ to the full $\mathrm{SU}(4)_{T}$ group. The quark bilinears we use ${ }^{3}$ transform in the 15 (adjoint) irrep of $\mathrm{SU}(4)_{T}$. The decomposition of the 15 irrep into $\mathrm{SU}(2)_{\mathbb{Q}_{8}} \times \mathrm{SU}(2)_{\mathrm{D}_{4}}$ irreps occurs via

$$
15 \rightarrow(1,1) \oplus(1,0) \oplus(0,1) .
$$

Just as above, the quantum numbers of $\mathrm{SU}(2)_{\mathbb{Q}_{8}} \times \mathrm{SU}(2)_{\mathrm{D}_{4}}$ can label the 15 irrep of $\mathrm{SU}(4)_{T}$ as there are no degenerate irreps in Eq. (A17). It should be noted that on the lattice, bosonic irreps can be classified according to a subgroup of the GTS group called the $\overline{\mathrm{RF}}$ group [25].

We will first examine the continuum quantum numbers of the local lattice vector current, $V$. At zero momentum, it has spin taste $\gamma_{4} \otimes \xi_{4}$. Within the $\overline{\mathrm{RF}}$ group, $V$ transforms as the trivial irrep, 1 [25]. We can decompose $\overline{\mathrm{RF}}$ into the discrete rotational subgroup, $\mathrm{SW}_{3}$, to get

$$
1 \rightarrow A_{1}
$$

where $A_{1}$ is the trivial irrep of $\mathrm{SW}_{3}$.

We denote as $\mathcal{V}$ the continuum operator corresponding to $V$ and apply the same subduction procedure as in the previous session by following the subgroup chain $\mathrm{SU}(2)_{S} \times \mathrm{SU}(2)_{\mathbb{Q}_{8}} \rightarrow \mathrm{SU}(2)_{\mathrm{SW}_{3}} \rightarrow \mathrm{SW}_{3}$. The spin-0 irrep

\footnotetext{
${ }^{3}$ We do not use the taste-scalar current as it is a multilink operator, which has been empirically observed to have more noise.
}

of $\mathrm{SU}(2)_{\mathrm{SW}_{3}}$ subduces into the trivial irrep of $\mathrm{SW}_{3}$ [62]. Consequently, $\mathcal{V}$ needs to be in the trivial irrep of $\mathrm{SU}(2)_{S} \times \mathrm{SU}(2)_{\mathbb{Q}_{8}}$, and matching $\mathbb{Q}_{8}$ factors, $\mathcal{V}$ can only transform as $(0,1)$ irrep of $\mathrm{SU}(2)_{\mathfrak{Q}_{8}} \times \mathrm{SU}(2)_{\mathrm{D}_{4}}$ from Eq. (A17).

We have just found that $\mathcal{V}$ is a triplet of $\mathrm{SU}(2)_{\mathrm{D}_{4}}$, and so we need to determine its $z$-component quantum number. With positive parity, the three $m^{\mathrm{D}_{4}}$ components of the $(0,0,1)$ irrep from $\mathrm{SU}(2)_{S} \times \mathrm{SU}(2)_{\mathbb{Q}_{8}} \times \mathrm{SU}(2)_{\mathrm{D}_{4}}$ subduce into the lattice currents $\gamma_{4} \otimes \gamma_{4}, \gamma_{4} \otimes \xi_{4} \xi_{5}$, and $\gamma_{4} \otimes \xi_{5}$. Each transforms trivially in $\overline{\mathrm{RF}}$. The first lattice current is local, and the other two are nonlocal with multilink connections between the quarks and antiquarks. The eigenvalues of $I_{S}$ are +1 for the local current and -1 for the other two. As discussed in Eq. (A8), the matrix representation of $I_{S}$ in the continuum can be constructed from the tensor product of representations of $\mathrm{SU}(2)_{\mathrm{D}_{4}}$, $U(1)_{\mathrm{D}_{4}}$, and $P$ to give

$e^{i \pi \times \operatorname{diag}(1,0,-1)} \otimes 1 \otimes 1=\left[\begin{array}{ccc}-1 & 0 & 0 \\ 0 & 1 & 0 \\ 0 & 0 & -1\end{array}\right]=I_{S}$,

where the $\mathrm{SU}(2)_{\mathrm{D}_{4}}$ factor is in a spin triplet as discussed, $U(1)_{\mathrm{D}_{4}}$ is a trivial factor to give the correct $I_{S}$ eigenvalues, and the parity is also trivial by construction. Consequently, to get the correct $I_{S}=1$ eigenvalue on the lattice, the local $\gamma_{4} \otimes \gamma_{4}$ current must have zero $z$ component in the triplet irrep of $\mathrm{SU}(2)_{\mathrm{D}_{4}}$ in the continuum limit. This completes the subduction of $\mathcal{V}$ into $V$.

The procedure is similar subducing the continuum axialvector current $\mathcal{A}$ into the lattice version $A$. On the lattice, $A$ transforms as a three-dimensional irrep, $3^{\prime \prime \prime \prime}$, of $\overline{\mathrm{RF}}$, which decomposes into the

$$
3^{\prime \prime \prime \prime} \rightarrow A_{1} \oplus E
$$

irreps of $\mathrm{SW}_{3}$. The linear combination

$A_{1} \propto\left(\gamma_{x} \gamma_{5} \otimes \xi_{x} \xi_{5}\right)+\left(\gamma_{y} \gamma_{5} \otimes \xi_{y} \xi_{5}\right)+\left(\gamma_{z} \gamma_{5} \otimes \xi_{z} \xi_{5}\right)$

transforms trivially under discrete rotations so it lives in the $A_{1}$ irrep. The remaining linear combinations are

$$
E_{+} \propto\left(\gamma_{x} \gamma_{5} \otimes \xi_{x} \xi_{5}\right)+\left(\gamma_{y} \gamma_{5} \otimes \xi_{y} \xi_{5}\right)-2\left(\gamma_{z} \gamma_{5} \otimes \xi_{z} \xi_{5}\right)
$$

$$
E_{-} \propto\left(\gamma_{x} \gamma_{5} \otimes \xi_{x} \xi_{5}\right)-\left(\gamma_{y} \gamma_{5} \otimes \xi_{y} \xi_{5}\right)
$$

where the subscript on the left-hand side is the eigenvalue \pm of $R_{12}$.

In the continuum, $\mathcal{A}$ is a spin- 1 operator of $\mathrm{SU}(2)_{S}$. The $A_{1}$ irrep subduces from the spin-0 irrep of $\mathrm{SU}(2)_{\mathrm{SW}_{3}}$, and 
the $E$ irrep subduces from the spin-2 irrep of $\mathrm{SU}(2)_{\mathrm{SW}_{3}}$. With the rules for the addition of angular momentum, this requires $\mathcal{A}$ to be in the irrep $(1,1)$ of $\mathrm{SU}(2)_{S} \times \mathrm{SU}(2)_{\mathbb{Q}_{8}}$ with zero $z$-component spins in both $\mathrm{SU}(2)$ factors.

Now, according to Eq. (A17), $\mathcal{A}$ can be either a spin-0 or 1 operator of $\mathrm{SU}(2)_{\mathrm{D}_{4}}$. Recall that on the lattice, $\mathrm{D}_{4}$ is generated by the transformations $I_{S}$ and $\Xi_{123}$ [20]. $A$ is an eigenvector of both these symmetries with respective eigenvalues 1 and -1 . Because $\mathrm{SU}(2)_{\mathrm{D}_{4}}$ subduces into the $\mathrm{D}_{4}$ factor of the GTS group, these nontrivial eigenvalues mean that $\mathcal{A}$ cannot transform trivially under $\mathrm{SU}(2)_{\mathrm{D}_{4}}$. As such, $\mathcal{A}$ can only belong to spin-1 irrep of $\mathrm{SU}(2)_{\mathrm{D}_{4}}$. Further, it has zero $z$ component following the same argument in Eq. (A19).

In summary, we have determined the continuum quantum numbers of $\mathcal{A}$ and $\mathcal{V}$, which subduce into the desired lattice current operators, $A$ and $V$, respectively. Using the same notation as in Eq. (A7), the continuum currents transform as

$$
\begin{gathered}
-\mathcal{A}_{(1,0)_{Q}(1,0)_{\mathrm{D}_{4}}}^{(1,0)_{S}(1,0)_{F}} \equiv \mathcal{A} / \sqrt{n_{t}} \rightarrow A / \sqrt{n_{t}}, \\
\mathcal{V}_{(0,0)_{\mathbb{Q}}(1,0)_{\mathrm{D}_{4}}}^{(0,0)_{S}(1,0)_{F}} \equiv \mathcal{V} / \sqrt{n_{t}} \rightarrow V / \sqrt{n_{t}} .
\end{gathered}
$$

The spin and flavor quantum numbers of the tensor operators are denoted by the superscripts, whereas the taste quantum numbers are given in the subscripts. $n_{t}=4$ is the number of tastes, and $\sqrt{n_{t}}=2$ is required to properly normalize tensor operators. The minus sign in front of the axial current is a convention that we follow according to Table I of Ref. [61].

As an aside, there is an easy way to obtain the continuum taste quantum numbers of an arbitrary quark bilinear without explicit group subduction. Table I of Ref. [61] outlines the SU(4) generators and their corresponding tensor operators. Once we adopt the Euclidean Dirac representation for the taste gamma matrices $\xi_{4}=\sigma_{3} \otimes I$, $\xi_{j}=\sigma_{2} \otimes \sigma_{j}$ (where $\sigma_{j}$ are the usual Pauli matrices), those generators give the components of the continuum taste matrices. For example, the local axial and vector currents we use have taste gamma matrices of

$$
\begin{aligned}
& \xi_{z} \xi_{5}=\left[\begin{array}{cccc}
1 & 0 & 0 & 0 \\
0 & -1 & 0 & 0 \\
0 & 0 & -1 & 0 \\
0 & 0 & 0 & 1
\end{array}\right], \\
& \xi_{4}=\left[\begin{array}{cccc}
1 & 0 & 0 & 0 \\
0 & 1 & 0 & 0 \\
0 & 0 & -1 & 0 \\
0 & 0 & 0 & -1
\end{array}\right]
\end{aligned}
$$

They are proportional to the generators $\frac{1}{2}\left(A_{11}-A_{22}-\right.$ $\left.A_{33}+A_{44}\right)$ and $\frac{1}{2}\left(A_{11}+A_{22}-A_{33}-A_{44}\right)$. By identifying $S$ as $\mathrm{D}_{4}$ in Table I of Ref. [61], and similarly $T$ as $\mathbb{Q}_{8}$, we can recognize the tensor product $S \otimes T=\sigma_{3} \otimes \sigma_{3}$ and $\sigma_{3} \otimes I$, indicating a spin-1 representation whenever a $\sigma_{3}$ appears in the tensor product. This yields the continuum taste quantum numbers of these states as $(1,0)_{\mathbb{Q}_{8}}(1,0)_{\mathrm{D}_{4}}$ and $(0,0)_{\mathbb{Q}_{8}}(1,0)_{\mathrm{D}_{4}} \cdot{ }^{4}$

\section{APPENDIX B: WIGNER-ECKART THEOREM AND THE PHYSICAL MATRIX ELEMENTS}

In this Appendix we need to relate, for each current, the $s= \pm 0$ nucleonlike lattice matrix elements to their physical continuum counterpart. We label the continuum matrix elements as

$$
\begin{aligned}
& M_{ \pm}^{V} \equiv\langle 16, \pm \overrightarrow{0}|\mathcal{V}| 16, \pm \overrightarrow{0}\rangle \\
& M_{ \pm}^{A} \equiv\langle 16, \pm \overrightarrow{0}|\mathcal{A}| 16, \pm \overrightarrow{0}\rangle
\end{aligned}
$$

Since we know the continuum quantum numbers of each state and current, we can apply the Wigner-Eckart theorem to relate the different components. To further reduce the number of independent matrix elements from four to two, we apply the Wigner-Eckart theorem to the $\mathrm{SU}(2)_{\mathbb{Q}_{8}}$ part of the irreps in Eqs. (A15), (A16), (A24), and (A25) to find

$$
\begin{gathered}
M_{-}^{A}=-3 M_{+}^{A}, \\
M_{-}^{V}=M_{+}^{V} .
\end{gathered}
$$

This result is consistent with the discussion around Appendix A 1. On the lattice, we have found exact symmetries for the local vector currents

$$
\begin{aligned}
\langle & \left\langle\left[\frac{3}{2}, \frac{3}{2}\right]_{F}[16,+\overrightarrow{0}]_{\mathrm{GTS}}|V|\left[\frac{3}{2}, \frac{3}{2}\right]_{F}[16,+\overrightarrow{0}]_{\mathrm{GTS}}\right\rangle \\
& =\left\langle\left[\frac{3}{2}, \frac{3}{2}\right]_{F}[16,-\overrightarrow{0}]_{\mathrm{GTS}}|V|\left[\frac{3}{2}, \frac{3}{2}\right]_{F}[16,-\overrightarrow{0}]_{\mathrm{GTS}}\right\rangle,
\end{aligned}
$$

which come from Eq. (A3) and

$$
\begin{aligned}
\sum_{\vec{x}, \vec{y}}\left\langle B_{+\overrightarrow{0}}^{16}(\vec{x}, t) V(\vec{y}, \tau) \bar{B}_{+\overrightarrow{0}}^{16}(0)\right\rangle \\
\quad=\sum_{\vec{x}, \vec{y}}\left\langle B_{-\overrightarrow{0}}^{16}(\vec{x}, t) V(\vec{y}, \tau) \bar{B}_{-\overrightarrow{0}}^{16}(0)\right\rangle,
\end{aligned}
$$

\footnotetext{
${ }^{4}$ There is a typo in Table I of Ref. [61]. The irreducible tensor components at line 3 should read $-T_{(1,0)(1,0)}^{[211]}$ instead of $-T_{(0,0)(0,0)}^{[211]}$.
} 

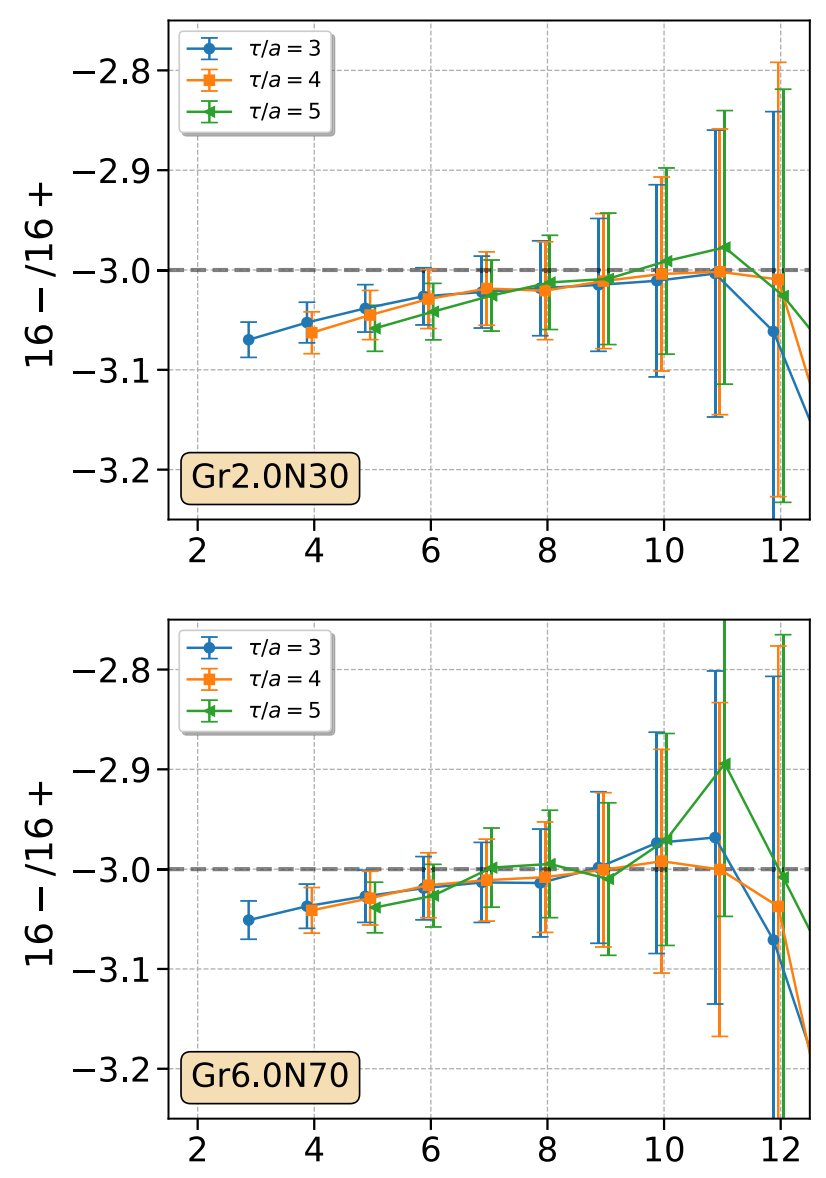

FIG. 9. The ratio of the three-point $g_{A}$ correlators, built with $\sum_{\vec{x}} B_{-\overrightarrow{0}}^{16}(\vec{x}, t)$ ("16-" with eigenvalues of -1 for the $x-y$ plane rotation) and $\sum_{\vec{x}} B_{+\overrightarrow{0}}^{16}(\vec{x}, t)$ ("16+" with eigenvalue of +1 ) interpolating operators, as a function of source-sink separation time $t$ and current insertion time $\tau$. Both interpolators are used in Eq. (2.4) to compute $g_{A}$. Each plot represents a different Wuppertal smearing at the sink, with parameters 0.2 (Gr2.0N30) and $0.6 \mathrm{fm}$ (Gr6.0N70) rms radii. The group theory requires that in the continuum and $t, \tau \rightarrow \infty$ limits that the ratio is equal to -3 , which is shown as dashed lines.

derived from applying lattice rotations and shifts. For the local axial-vector current, there are no symmetries relating the matrix elements on the lattice, but the relationship in Eq. (B3) emerges in the continuum.

To demonstrate this observation, we have plotted the ratio of optimized $g_{A}$ three-point correlators created with $\sum_{\vec{x}} B_{-\overrightarrow{0}}^{16}(\vec{x}, t)$ and $\sum_{\vec{x}} B_{+\overrightarrow{0}}^{16}(\vec{x}, t)$ interpolators in Fig. 9. In the limits $\tau, t-\tau \rightarrow \infty$ and $a \rightarrow 0$, the ratio should converge to the dashed lines at -3 as predicted by the above group theory. The small deviation is caused by a combination of excited-state contamination, discretization effects, and taste-breaking effects. The same ratio for the vector current is consistent with one to high precision, as enforced by the lattice relation in Eq. (B6). Figure 9 is therefore a nontrivial verification of our group theory understanding of staggered baryon matrix elements.
To relate the two remaining staggered matrix elements to their counterparts in QCD without tastes we observe that

$$
\begin{aligned}
& \left|M_{\text {phy }}^{V}\right|=|\langle B|\mathcal{V}| B\rangle|, \\
& \left|M_{\text {phy }}^{A}\right|=|\langle B|\mathcal{A}| B\rangle|,
\end{aligned}
$$

where $M_{\text {phy }}^{V}$ and $M_{\text {phy }}^{A}$ are the physical vector and axial matrix elements. Here

$$
|B\rangle \equiv\left|\left[\frac{1}{2}, \frac{1}{2}\right]_{S}\left[\frac{1}{2}, \frac{1}{2}\right]_{F}\left[\frac{3}{2}, \frac{3}{2}\right]_{\mathbb{Q}_{8}}\left[\frac{3}{2}, \frac{3}{2}\right]_{\mathrm{D}_{4}}\right\rangle
$$

is the single-taste nucleon; e.g., $|B\rangle$ has the correct isospin of $\frac{1}{2}$ and transforms as the symmetric $20_{S}$ irrep of $\mathrm{SU}(4)_{T}$. The $20_{S}$ irrep of $\mathrm{SU}(4)_{T}$ contains states with a single-taste baryon. ${ }^{5}$ To relate single-taste baryon matrix elements to the physical one, we also need the taste-diagonal current operators which have tastes $\xi_{z} \xi_{5}, \xi_{4}, \xi_{1} \xi_{2}$, or $\mathbf{1}$. These constructions must coincide with the physical matrix elements, up to a sign, if the taste restoration is valid in the continuum limit.

Again, we can use the quantum numbers of $\mathrm{SU}(2)_{\mathbb{Q}_{8}} \times$ $\mathrm{SU}(2)_{\mathrm{D}_{4}}$ to uniquely label components in $20_{S}$ because there are no degenerate irreps in the decomposition $20_{S} \rightarrow\left(\frac{3}{2}, \frac{3}{2}\right) \oplus\left(\frac{1}{2}, \frac{1}{2}\right)$. We apply the Wigner-Eckart theorem to normalize the matrix elements, $M_{-}^{V}$ and $M_{-}^{A}$, to $M_{\text {phy }}^{V}$ and $M_{\text {phy }}^{A}$. This boils down to finding the correct ClebschGordon coefficients to rotate $|16,-\overrightarrow{0}\rangle$ to the single-taste baryon $|B\rangle$ while leaving the taste-diagonal currents unchanged. An $\mathrm{SU}(4)_{T}$ rotation alone is insufficient because these states belong to different $\mathrm{SU}(4)_{T}$ irreps. However, we can embed flavor and taste into a larger group and perform rotations in this larger group to accomplish the task. If we consider the relevant group factors $\mathrm{SU}(4)_{F \times \mathrm{D}_{4}} \supset \mathrm{SU}(2)_{F} \times \mathrm{SU}(2)_{\mathrm{D}_{4}}$, both $|16,-\overrightarrow{0}\rangle$ and $|B\rangle$ belong to the same $20_{M}$ irrep of SU $(4)_{F \times D_{4}}$, and so we can apply the Wigner-Eckart theorem to this group.

The details of the generalized Wigner-Eckart theorem for SU(4) are described in Ref. [61]. We will only need the Wigner-Eckart theorem in Eq. (33) of that reference, and the Clebsch-Gordon coefficients in Table A4.5 of Ref. [61], to conclude that

$$
\begin{aligned}
& \left|M_{-}^{V}\right|=|\langle B|\mathcal{V}| B\rangle|=\left|M_{\text {phy }}^{V}\right|, \\
& \left|M_{-}^{A}\right|=|\langle B|\mathcal{A}| B\rangle|=\left|M_{\text {phy }}^{A}\right| .
\end{aligned}
$$

We can understand the trivial normalization factor by realizing that in the continuum, $\mathrm{SU}(2)_{F}, \mathrm{SU}(2)_{\mathbb{Q}_{8}}$, and

\footnotetext{
${ }^{5}$ As an analogy, the single taste of $\mathrm{SU}(2)_{T}$ is similar to the $\Delta^{++}$ (consisting of three valence up-quarks) in $\mathrm{SU}(2)_{F}$.
} 
$\mathrm{SU}(2)_{\mathrm{D}_{4}}$ are indistinguishable from one another because of the enlarged $\mathrm{SU}(8)_{\mathrm{FT}}$ symmetry. This means that the matrix elements are invariant under the exchange of $\mathrm{D}_{4}$ and $F$ labels in Eq. (B7) and Eq. (B8). This shows that Eq. (B7) and Eq. (B8) are identical to Eq. (B1) and Eq. (B2), and hence, the trivial normalization factors. Combining the shift symmetry relationship in the correlators from Eqs. (A4) with Eqs. (B11) gives a key result for this paper, which is presented in Eqs. (2.3) and (2.4).
[1] A. S. Kronfeld, D. G. Richards, W. Detmold, R. Gupta, H.-W. Lin, K.-F. Liu, A. S. Meyer, R. Sufian, and S. Syritsin (USQCD Collaboration), Eur. Phys. J. A 55, 196 (2019).

[2] R. R. de Austri and C. P. de los Heros, J. Cosmol. Astropart. Phys. 11 (2013) 049.

[3] L. Alvarez-Ruso et al. (NuSTEC Collaboration), Prog. Part. Nucl. Phys. 100, 1 (2018).

[4] J. Ellis, N. Nagata, and K. A. Olive, Eur. Phys. J. C 78, 569 (2018).

[5] J. Arrington, C. Roberts, and J. Zanotti, J. Phys. G 34, S23 (2007).

[6] Z. Ye, J. Arrington, R. J. Hill, and G. Lee, Phys. Lett. B 777, 8 (2018).

[7] G. Lee, J. R. Arrington, and R. J. Hill, Phys. Rev. D 92, 013013 (2015).

[8] R. J. Hill, EPJ Web Conf. 137, 01023 (2017).

[9] H.-W. Hammer and U.-G. Meißner, Sci. Bull. 65, 257 (2020).

[10] K. Borah, R. J. Hill, G. Lee, and O. Tomalak, Phys. Rev. D 102, 074012 (2020).

[11] A. S. Meyer, M. Betancourt, R. Gran, and R. J. Hill, Phys. Rev. D 93, 113015 (2016).

[12] P. Zyla et al. (Particle Data Group), Prog. Theor. Exp. Phys. 2020, 083C01 (2020).

[13] R. J. Hill, P. Kammel, W. J. Marciano, and A. Sirlin, Rep. Prog. Phys. 81, 096301 (2018).

[14] G. Parisi, Phys. Rep. 103, 203 (1984).

[15] G. P. Lepage, in From Actions to Answers, edited by T. DeGrand and D. Toussaint (World Scientific, Singapore, 1990), pp. 97-120.

[16] A. Bazavov et al. (MILC Collaboration), Phys. Rev. D 87, 054505 (2013).

[17] E. Follana, Q. Mason, C. Davies, K. Hornbostel, G. P. Lepage, J. Shigemitsu, H. Trottier, and K. Wong (HPQCD Collaboration), Phys. Rev. D 75, 054502 (2007).

[18] M. F. L. Golterman and J. Smit, Nucl. Phys. B255, 328 (1985).

[19] J. A. Bailey, Phys. Rev. D 75, 114505 (2007).

[20] Y. Lin, A. S. Meyer, C. Hughes, A. S. Kronfeld, J. N. Simone, and A. Strelchenko, Phys. Rev. D 103, 034501 (2021).

[21] J. A. Bailey et al., Phys. Rev. D 79, 054507 (2009).

[22] B. J. Owen, J. Dragos, W. Kamleh, D. B. Leinweber, M. Mahbub, B. J. Menadue, and J. M. Zanotti, Phys. Lett. B 723, 217 (2013).

[23] E. Berkowitz et al., arXiv:1704.01114; C. Chang et al., Nature (London) 558, 91 (2018).
[24] R. Gupta, Y.-C. Jang, B. Yoon, H.-W. Lin, V. Cirigliano, and T. Bhattacharya, Phys. Rev. D 98, 034503 (2018).

[25] M. F. Golterman, Nucl. Phys. B273, 663 (1986).

[26] Z. Gelzer et al. (Fermilab Lattice and MILC Collaborations), Proc. Sci., LATTICE2019 (2019) 236 [arXiv:1912.13358].

[27] C. Michael and I. Teasdale, Nucl. Phys. B215, 433 (1983); A. S. Kronfeld, Nucl. Phys. B, Proc. Suppl. 17, 313 (1990); M. Lüscher and U. Wolff, Nucl. Phys. B339, 222 (1990).

[28] A. Hart, G. von Hippel, and R. Horgan (HPQCD Collaboration), Phys. Rev. D 79, 074008 (2009).

[29] A. Bazavov et al., Phys. Rev. D 98, 074512 (2018).

[30] O. Ledoit and M. Wolf, Ann. Stat. 40, 1024 (2012).

[31] S. Güsken, Nucl. Phys. B, Proc. Suppl. 17, 361 (1990).

[32] G. S. Bali, B. Lang, B. U. Musch, and A. Schäfer, Phys. Rev. D 93, 094515 (2016).

[33] G. P. Lepage, gplepage/corrfitter: Corrfitter version 8.1 (2020).

[34] A. Walker-Loud, Proc. Sci., LATTICE2013 (2014) 013 [arXiv:1401.8259].

[35] S. Aoki et al. (Flavour Lattice Averaging Group), Eur. Phys. J. C 80, 113 (2020).

[36] C. Alexandrou, S. Bacchio, M. Constantinou, J. Finkenrath, K. Hadjiyiannakou, K. Jansen, G. Koutsou, and A. Vaquero Aviles-Casco, Phys. Rev. D 102, 054517 (2020).

[37] G. S. Bali, L. Barca, S. Collins, M. Gruber, M. Löffler, A. Schäfer, W. Söldner, P. Wein, S. Weishäupl, and T. Wurm (RQCD Collaboration), J. High Energy Phys. 05 (2020) 126.

[38] J. Liang, Y.-B. Yang, T. Draper, M. Gong, and K.-F. Liu, Phys. Rev. D 98, 074505 (2018).

[39] T. Harris, G. von Hippel, P. Junnarkar, H. B. Meyer, K. Ottnad, J. Wilhelm, H. Wittig, and L. Wrang, Phys. Rev. D 100, 034513 (2019).

[40] K.-I. Ishikawa, Y. Kuramashi, S. Sasaki, N. Tsukamoto, A. Ukawa, and T. Yamazaki (PACS Collaboration), Phys. Rev. D 98, 074510 (2018).

[41] N. Yamanaka, S. Hashimoto, T. Kaneko, and H. Ohki (JLQCD Collaboration), Phys. Rev. D 98, 054516 (2018).

[42] J. Green, M. Engelhardt, S. Krieg, J. Negele, A. Pochinsky, and S. Syritsyn, Phys. Lett. B 734, 290 (2014).

[43] J. Bratt et al. (LHP Collaboration), Phys. Rev. D 82, 094502 (2010).

[44] T. Yamazaki, Y. Aoki, T. Blum, H. Lin, M. Lin, S. Ohta, S. Sasaki, R. Tweedie, and J. Zanotti (RBC+UKQCD Collaboration), Phys. Rev. Lett. 100, 171602 (2008).

[45] T. Yamazaki, Y. Aoki, T. Blum, H.-W. Lin, S. Ohta, S. Sasaki, R. Tweedie, and J. Zanotti, Phys. Rev. D 79, 114505 (2009). 
[46] R. Edwards, G. Fleming, P. Hagler, J. Negele, K. Orginos, A. Pochinsky, D. Renner, D. Richards, and W. Schroers (LHPC Collaboration), Phys. Rev. Lett. 96, 052001 (2006).

[47] S. Capitani, M. Della Morte, D. Djukanovic, G. M. von Hippel, J. Hua, B. Jager, P. M. Junnarkar, H. B. Meyer, T. D. Rae, and H. Wittig, Int. J. Mod. Phys. A 34, 1950009 (2019).

[48] C. Alexandrou, M. Constantinou, K. Hadjiyiannakou, K. Jansen, C. Kallidonis, G. Koutsou, and A. Vaquero AvilesCasco, Phys. Rev. D 96, 054507 (2017).

[49] A. Abdel-Rehim et al., Phys. Rev. D 92, 114513 (2015); 93, 039904(E) (2016).

[50] G. S. Bali, S. Collins, B. Glässle, M. Göckeler, J. Najjar, R. H. Rödl, A. Schäfer, R. W. Schiel, W. Söldner, and A. Sternbeck, Phys. Rev. D 91, 054501 (2015).

[51] R. Horsley, Y. Nakamura, A. Nobile, P. Rakow, G. Schierholz, and J. Zanotti, Phys. Lett. B 732, 41 (2014).

[52] S. Capitani, M. Della Morte, G. von Hippel, B. Jager, A. Juttner, B. Knippschild, H. Meyer, and H. Wittig, Phys. Rev. D 86, 074502 (2012).
[53] H.-W. Lin, T. Blum, S. Ohta, S. Sasaki, and T. Yamazaki, Phys. Rev. D 78, 014505 (2008).

[54] A. Khan et al., Phys. Rev. D 74, 094508 (2006).

[55] G. S. Bali, S. Collins, and A. Schäfer, Comput. Phys. Commun. 181, 1570 (2010).

[56] T. Blum, T. Izubuchi, and E. Shintani, Phys. Rev. D 88, 094503 (2013).

[57] M. Lüscher, J. High Energy Phys. 08 (2010) 071; 03 (2014) 92.

[58] C. C. Chang et al., EPJ Web Conf. 175, 01008 (2018).

[59] A. S. Meyer, The nucleon axial form factor and staggered lattice QCD, Ph.D. thesis, University of Chicago, 2017.

[60] C. W. Bernard et al. (MILC Collaboration), Phys. Rev. D 58, 014503 (1998).

[61] K. T. Hecht and S. C. Pang, J. Math. Phys. (N.Y.) 10, 1571 (1969).

[62] S. Basak, R. Edwards, G. T. Fleming, U. M. Heller, C. Morningstar, D. Richards, I. Sato, and S. J. Wallace (Lattice Hadron Physics Collaboration), Phys. Rev. D 72, 074501 (2005). 